TRANSACTIONS OF THE

AMERICAN MATHEMATICAL SOCIETY

Volume 232, 1977

\title{
VECTOR FIELDS ON POLYHEDRA
}

\author{
BY
}

Michael A. PENNA

\begin{abstract}
This paper presents a bundle theory for studying vector fields and their integral flows on polyhedra ${ }_{*}$ and applications.

Every polyhedron has a tangent object in the category of simplicial bundles in much the same way as every smooth manifold has a tangent object in the category of smooth vector bundles. One can show that there is a correspondence between piecewise smooth flows on a polyhedron $P$ and sections of the tangent object of $P$ (i.e., vector fields on $P$ ); using this result one can prove existence results for piecewise smooth flows on polyhedra. Finally an integral formula for the Euler characteristic of a closed, oriented, even-dimensional combinatorial manifold is given; as a consequence of this result one obtains a representation of Euler classes of such combinatorial manifolds in terms of piecewise smooth forms.
\end{abstract}

0. Introduction. The purpose of this paper is to describe a bundle theory for studying vector fields and their integral flows on polyhedra and, in particular, on combinatorial manifolds. Two applications of this theory are presented; these results are motivated by corresponding results for smooth manifolds.

Here are the results obtained:

THEOREM A. The sum of the indices of any integrable vector field on a polyhedron $P$ which has a finite number of zeroes is the Euler characteristic $\chi(P)$ of $P$. Furthermore, if $M$ is a closed combinatorial manifold with $\chi(M)=0$, then there is a nowhere vanishing integrable vector field on $M$.

Theorem A corresponds to a classical result for smooth manifolds due to Hopf (see [10]). Actually Hopf's original result (see [7]) is also valid for a particular type of vector field on a combinatorial manifold. It will be seen that even though there are similarities between Hopf's original result and Theorem A, there are also major differences. As a special case of Theorem A one also obtains an analog to a calculation of Whitney (see [14]) giving the Euler characteristic of a triangulated smooth manifold using the singularities of vector fields.

Now let $M$ be a closed, oriented, $2 n$-dimensional combinatorial manifold.

Received by the editors February 28, 1975 and, in revised form, March 15, 1976.

AMS (MOS) subject classifications (1970). Primary 58A99, 57B99, 57D25.

Key words and phrases. Polyhedron, manifold, tangent bundle, vector field, Hopf's theorem, Gauss-Bonnet formula.

- American Mathematical Society 1977 
From the proof of Theorem A it follows that there is an integrable vector field $s$ on $M$ with a finite number of zeroes interior to the $2 n$-simplices $\sigma_{\alpha}^{2 n}$ of $M$. On each $\sigma_{\alpha}^{2 n}$ one can define smooth forms $e_{\alpha}^{2 n} \in \Lambda^{2 n} E\left(\sigma_{\alpha}^{2 n}\right)$ and $\eta_{\alpha}^{2 n-1}$ $\in \Lambda^{2 n-1} E\left(T \sigma_{\alpha}^{2 n}\right)$ for which

$$
\int_{\sigma_{\alpha}^{2 n}} e_{\alpha}^{2 n}-\int_{\partial \sigma_{\alpha}^{2 n}} s^{*} \eta_{\alpha}^{2 n-1}=\sum i\left(s, x_{k}\right)
$$

where $i\left(s, x_{k}\right)$ is the index of $s$ at the zero $x_{k}$ of $s$, and the summation is taken over all zeroes $x_{k} \in \sigma_{\alpha}^{2 n}$.

THEOREM B. On a closed, oriented, $2 n$-dimensional combinatorial manifold $M$ the forms $e_{\alpha}^{2 n}-d s^{*} \eta_{\alpha}^{2 n-1}$, for $\sigma_{\alpha}^{2 n}$ a $2 n$-simplex of $M$ and $s$ an integrable vector field on $M$, define a global closed piecewise smooth 2 -form $e(M) \in \Lambda^{2 n} E(M)$ on $M$, and $\int_{M} e(M)=\chi(M)$.

Theorem B is closely related to a result of Allendoerfer and Weil (see [1]) which gives a similar integral formula for the Euler characteristic of a smooth manifold with piecewise smooth boundary. The proof of Theorem B is similar to Chern's intrinsic proof of the Gauss-Bonnet formula for closed Riemannian manifolds (see [5]).

The bundle theory which yields these results is the theory of simplicial bundles. There are two major reasons why this theory is important in the study of polyhedral differential geometry. First this theory helps relate piecewise smooth forms on a polyhedron with vector fields, and more specifically piecewise smooth flows, on a polyhedron. Second, in analogy with smooth manifolds, this theory illustrates a global differential geometry on polyhedra which arises naturally from local constructions on "standard" open sets (small open sets) which can be coordinatized. This global theory is furthermore intrinsic: The differential geometry of a polyhedron in a neighborhood of a point depends only on what that neighborhood looks like.

The theory of simplicial bundles is the dual of a corresponding bundle theory which is used in studying piecewise smooth forms on polyhedra (see [11]); some of the terminology and results of [11] are briefly reviewed in $\$ 1$. Simplicial bundles are defined in \$2: every polyhedron has a tangent object in the category of simplicial bundles in much the same way every smooth manifold has a tangent object in the category of smooth vector bundles. In $\$ 3$ piecewise smooth flows are defined, and the relation between piecewise smooth flows and integrable sections of the tangent object is described; using this relation one can prove existence results for piecewise smooth flows. In §4, Theorem $A$ is proved. In $\$ 5$, Riemannian metrics on polyhedra are considered. Finally in $\$ 6$, Theorem B is proved. 
1. Simplicial spaces. This section is devoted to a brief review of some of the terminology and results of [11].

For $n=0,1,2, \ldots$, the $n$-corner $s^{n}=\left\{x=\left(x_{k}\right) \in R^{n}: x_{k} \geqslant 0 \forall k\right\}$.

1.1. Definition. A simplicial chart on the topological space $X$ is a pair $(U, \psi)$ consisting of a nonempty subset (not necessarily an open subset) $U$ of $X$ and a homeomorphism $\psi: U \rightarrow V$ where $V$ is an open subset of some $n$-corner $s^{n}$. Two simplicial charts $\psi_{\alpha}: U_{\alpha} \rightarrow V_{\alpha} \subseteq s_{\alpha}^{m}$ and $\psi_{\beta}: U_{\beta} \rightarrow V_{\beta} \subseteq s_{\beta}^{n}$ are related iff $\psi_{\alpha}\left(U_{\alpha} \cap U_{\beta}\right)$ and $\psi_{\beta}\left(U_{\alpha} \cap U_{\beta}\right)$ are open subsets of $k$-faces of $s_{\alpha}^{m}$ and $s_{\beta}^{n}$, for some $k$ depending on $U_{\alpha}$ and $U_{\beta}$, and for which

$$
\psi_{\beta} \circ \psi_{\alpha}^{-1}: \psi_{\alpha}\left(U_{\alpha} \cap U_{\beta}\right) \rightarrow \psi_{\beta}\left(U_{\alpha} \cap U_{\beta}\right)
$$

is an invertible affine transformation (both domain and range being restricted). A simplicial atlas on $X$ is a collection of related simplicial charts on $X$ whose domains cover $X$ and which is maximal with respect to the inclusion of related simplicial charts. A simplicial space $X$ is a separable Hausdorff topological space equipped with a locally finite, finite-dimensional simplicial atlas.

Observe that in the definition of "related", $m$ and $n$ are not required to be equal, but $k$ is required to be the dimension of both $\psi_{\alpha}\left(U_{\alpha} \cap U_{\beta}\right)$ and $\psi_{\beta}\left(U_{\alpha} \cap U_{\beta}\right)$.

The main examples of simplicial spaces considered in this paper are polyhedra, i.e., locally finite, finite-dimensional simplicial complexes.

A simplicial space is a space equipped with an atlas of related simplicial charts, and different atlases on a space determine different simplicial spaces. There is, for example, a natural atlas on $s^{n}$, for $n \geqslant 1$, generated by the identity id: $s^{n} \rightarrow s^{n}$, and the resulting simplicial space is different than any simplicial space determined by a triangulation of $s^{n}$. (Similar remarks hold for $R^{n}$, for $n \geqslant 1$, and many CW complexes.) Thus the category of polyhedra can be considered as a full subcategory of the category of simplicial spaces.

Many of the following results also hold for triangulable spaces and, in particular, for PL manifolds as a consequence of naturality of the appropriate constructions with respect to subdivision. In this paper, however, we consider only simplicial spaces since these spaces are in many ways more natural to use.

A simplicial space $X$ may be described equivalently as a topological space $X$ equipped with an atlas of related, locally finite, finite dimensional "packets" on $X$.

1.2. Definition. A packet on a topological space $X$ is a pair $(U, \Psi)$ consisting of a nonempty open subset $U$ of $X$ and a collection $\Psi$ of related simplicial charts $\left(U_{\alpha}, \psi_{\alpha}\right)$ on $X, \alpha$ in some finite index set $A$, where $U$ $=\cup_{\alpha \in A} U_{\alpha}$ and for which:

(1) $U_{\alpha}$ is an open subset of $U_{\beta}$ iff $\alpha=\beta$, and 
(2) for $\alpha, \beta \in A$ there is a $\gamma \in A$ for which $U_{\gamma}=U_{\alpha} \cap U_{\beta}$.

The "domains" $U$ of packets $(U, \Psi)$ may be characterized intrinsically; such sets are called small open subsets: Let $x_{0} \in X$. For every simplicial chart $\psi_{\alpha}: U_{\alpha} \rightarrow V_{\alpha} \subseteq s_{\alpha}^{n}$ on $X$ for which $x_{0} \in U_{\alpha}, \psi_{\alpha}\left(x_{0}\right)$ is contained in the interior of a unique face $s_{\alpha}^{n}\left(x_{0}\right)$ of $s^{n}$.

1.3. Definition. An open neighborhood $U$ of $x_{0}$ is small iff $U$ may be written as the finite union of domains $U_{\alpha}$ of simplicial charts $\psi_{\alpha}: U_{\alpha} \rightarrow V_{\alpha}$ $\subseteq s_{\alpha}^{n}$ on $X, U_{\alpha}$ containing $x_{0}$, such that $V_{\alpha}$ meets no face of $s_{\alpha}^{n}$ of dimension less than or equal to that of $s_{\alpha}^{n}\left(x_{0}\right)$, except $s_{\alpha}^{n}\left(x_{0}\right)$ itself, of course. A small open subset $U$ of $X$ is an open subset of $X$ which is a small open neighborhood of some $x_{0} \in U$.

The set of small open subsets of a simplicial space $X$ forms a basis for the topology of $X$.

Every small open subset has a natural decomposition into "wedges".

1.4. Definimion. A wedge $U_{\alpha}$ of the small open subset $U$ is a subset of $U$ which is the domain of a simplicial chart $\left(U_{\alpha}, \psi_{\alpha}\right)$ in a packet structure $\Psi$ on $U$.

We now do analysis on simplicial spaces.

A smooth function $f: s^{n} \rightarrow R$ on the $n$-corner $s^{n}$ is a map which is smooth when restricted to the interior of each face of $s^{n}$ and such that for each $x_{0}$ on the boundary of $s^{n}$, the limit of $D f(x)$ as $x$ approaches $x_{0}$ is finite for every differential operator

$$
D=\partial^{q_{1}+\cdots+q_{n}} / \partial x_{1}^{q_{1}} \cdots \partial x_{n}^{q_{n}} .
$$

A smooth p-form on $s^{n}$ is any differential form $\sum f_{k} d f_{k_{1}} \cdots d f_{k_{p}}$ where $f_{k}, f_{k_{1}}$, $\ldots, f_{k_{p}}$ are smooth functions on $s^{n}$.

1.5. Definition. For $p=0,1,2, \ldots$ and any small open subset $U$ of the simplicial space $X$, the set $\Lambda^{p} E(U)$ of piecewise smooth p-forms on $U$ is the set of compatible tuples $\theta=\left(\theta_{\alpha}\right)$ of smooth $p$-forms $\theta_{\alpha}$ defined on the wedges $U_{\alpha}$ of $U$.

By "compatible" we mean that if $U_{\alpha}$ and $U_{\beta}$ are two wedges of $U$ then $\theta_{\alpha \mid U_{\alpha} \cap U_{\beta}}=\theta_{\beta \mid U_{\alpha} \cap U_{\beta}}$ where, for a smooth $p$-form $\theta=\sum f_{k} d f_{k_{1}} \cdots d f_{k_{p}}$ on $U_{\alpha}$,

$$
\theta_{\mid U_{\alpha} \cap U_{\beta}}=\Sigma\left(f_{k \mid U_{\alpha} \cap U_{\beta}}\right) d\left(f_{k_{1} \mid U_{\alpha} \cap U_{\beta}}\right) \cdots d\left(f_{k_{p} \mid U_{\alpha} \cap U_{\beta}}\right) \text {. }
$$

In particular $(p=0)$, the ring $A(U)$ of piecewise smooth functions on $U$ consists of those continuous functions $f: U \rightarrow R$ for which $f$ is smooth on each wedge of $U$. The set of piecewise smooth $p$-forms on $U$ becomes an $A(U)$ module by using wedgewise operations.

Wedgewise differentiation of piecewise smooth forms gives a cochain complex $\left(\Lambda^{*} E(U), d(U)\right)$. This construction is natural, and hence defines a cochain complex of presheaves $\left(\Lambda^{*} E(X), \underline{d}(X)\right)$ on $X$. 
1.6. Definition. The de Rham complex $\left(\Lambda^{*} E(X), d(X)\right)$ of piecewise smooth forms on the simplicial space $X$ is the cochain complex of global sections of the cochain complex of sheaves associated to $\left(\Lambda^{*} E(X), \underline{d}(X)\right)$.

Again, in particular $(p=0)$, the ring $A(X)$ of piecewise smooth functions on the simplicial space $X$ consists of all those continuous functions $f: X \rightarrow R$ for which $f \circ \psi^{-1}: V \rightarrow R$ is smooth on $V$ for every simplicial chart $\psi: U \rightarrow V$ on $X$. Furthermore the set of global piecewise smooth $p$-forms on $X$ naturally becomes an $A(X)$-module.

1.7. THEOREM (DE RHAM). The homology of the de Rham complex $\left(\Lambda^{*} E(X)\right.$, $d(X))$ of piecewise smooth forms on the simplicial space $X$ is isomorphic to the real singular cohomology of $X$.

It is not difficult to define the integral of a piecewise smooth form on a simplicial space. Integration yields a map from the graded module of piecewise smooth forms on a simplicial space $X$ to the graded module of singular simplicial cochains on $X$. Integration is a cochain map (Stokes's theorem), and hence induces a map in cohomology; de Rham's theorem states that this cohomology map is a ring isomorphism.

A cosimplicial bundle is a continuous surjection $\xi: E \rightarrow X$ from a topological space $E$ to a simplicial space $X$ for which $\xi^{-1}(x)$ is a finite-dimensional real vector space for each $x \in X$, and which satisfies certain other criteria. There is a category of cosimplicial bundles which contains a subcategory of vector bundles. In general, however, cosimplicial bundles differ from vector bundles since fiber dimensions in cosimplicial bundles are allowed to vary. $\mathbf{A}$ section $s$ of a cosimplicial bundle $\xi: E \rightarrow X$ is a (not necessarily continuous) function $s: X \rightarrow E$ for which $\xi \circ s=\mathrm{id}_{X}$ and which satisfies certain other criteria; the set $\Gamma(X, \xi)$ of global sections of $\xi$ forms an $A(X)$-module.

In much the same way every smooth manifold has a cotangent object in the category of smooth vector bundles, every simplicial space $X$ has a related cotangent object $\tau^{*}(X)$ in the category of cosimplicial bundles. The cosimplicial bundle $\tau^{*}(X)$ is a cotangent object for $X$ since there is an $A(X)$-module isomorphism between the module $E(X)$ of global piecewise smooth 1-forms on $X$ and the module $\Gamma\left(X, \tau^{*}(X)\right)$ of global sections of $\tau^{*}(X)$. Such cotangent objects are not usually vector bundles.

2. Simplicial bundles. The category of simplicial bundles is the dual of the category of cosimplicial bundles. Every simplicial space has a tangent object in the category of simplicial bundles in much the same way every smooth manifold has a tangent object in the category of smooth vector bundles.

Henceforth all vector spaces are finite-dimensional, real, and carry the usual topology.

2.1. Definition. A simplicial bundle is a continuous surjection $\xi: E \rightarrow X$ 
from a topological space $E$ to a simplicial space $X$ such that $\xi^{-1}(x)$ is a vector space for each $x \in X$, and:

(1) for every simplicial chart $\left(U_{\alpha}, \psi_{\alpha}\right)$ on $X$ there is a vector space $F_{\alpha}$ and a fiber preserving embedding $\phi_{\alpha}: U_{\alpha} \times F_{\alpha} \rightarrow E$ which is a linear injection on each fiber,

(2) if $\left(U_{\alpha}, \psi_{\alpha}\right)$ and $\left(U_{\beta}, \psi_{\beta}\right)$ are simplicial charts on $X$ for which $U_{\alpha} \subseteq U_{\beta}$, then $\phi_{\alpha}(x,-)=\phi_{\beta}(x,-) \circ l_{\beta \alpha}$ for every $x \in U_{\alpha}$ and some fixed linear embed$\operatorname{ding} l_{\beta \alpha}: F_{\alpha} \rightarrow F_{\beta}$,

(3) if $\left(U_{\alpha}, \psi_{\alpha}\right),\left(U_{\beta}, \psi_{\beta}\right)$, and $\left(U_{\gamma}, \psi_{\gamma}\right)$ are simplicial charts on $X$ for which $U_{\alpha} \subseteq U_{\beta} \subseteq U_{\gamma}$, then $l_{\gamma \alpha}=l_{\gamma \beta} \circ l_{\beta \alpha}$,

(4) if $\left(U_{\alpha}, \psi_{\alpha}\right)$ and $\left(U_{\beta}, \psi_{\beta}\right)$ are simplicial charts on $X$ for which $U_{\alpha}$ is an open subset of $U_{\beta}$, then $l_{\beta \alpha}$ is a linear isomorphism, and

(5) for each $x \in X, \xi^{-1}(x)$ is spanned by the set of all $\phi_{\alpha}(x,-) F_{\alpha}$ such that $\left(U_{\alpha}, \psi_{\alpha}\right)$ is a simplicial chart on $X$ and $x \in U_{\alpha}$.

Simplicial bundles and the appropriate morphisms form a category.

2.2. EXAMPLE. Let $X=P$ be the polyhedron which has three vertices $x_{0}, x_{1}$, and $x_{2}$, and which has two 1-simplices $\left(x_{0}, x_{1}\right)$ and $\left(x_{0}, x_{2}\right)$. Let $\pi_{1}: X \times R^{2}$ $\rightarrow X$ be the product vector bundle, $R^{2}$ being equipped with basis vectors $\underline{e}_{1}$ and $\underline{e}_{2}$. Let $E$ be the subspace of $X \times R^{2}$ consisting of all those $(x, v)$ for which $v$ is a multiple of $\underline{e}_{k}$ if $x \in\left(x_{0}, x_{k}\right)-\left\{x_{0}\right\}$, and $v \in R^{2}$ if $x=x_{0}$. If $\xi: E \rightarrow X$ is the map given by $\xi(x, v)=x$ for $(x, v) \in E$, then $\xi$ is a simplicial bundle. The structure fibers $F_{\alpha}$ of $\xi$ are as follows: $F_{\alpha}$ is the zero vector space $R^{0}$ if $U_{\alpha}=\left\{x_{0}\right\}$ and otherwise $F_{\alpha}$ is the vector space on one generator $\underline{e}_{k}$ whenever $U_{\alpha} \subseteq\left(x_{0}, x_{k}\right)$. The structure maps $\phi_{\alpha}: U_{\alpha} \times F_{\alpha} \rightarrow E$ of $\xi$ are as follows: $\phi_{\alpha}\left(x_{0}, 0\right)=\left(x_{0}, 0\right)$ if $U_{\alpha}=\left\{x_{0}\right\}$ and otherwise $\phi_{\alpha}\left(x, c \underline{e}_{k}\right)=\left(x, c \underline{e}_{k}\right)$ whenever $U_{\alpha} \subseteq\left(x_{0}, x_{k}\right)$. Notice that $\xi$ is clearly not a vector bundle since $\xi^{-1}\left(x_{0}\right)=R^{2}$ and $\xi^{-1}(x)=R^{1}$ for all other $x \in X$. (See Diagram 1.)

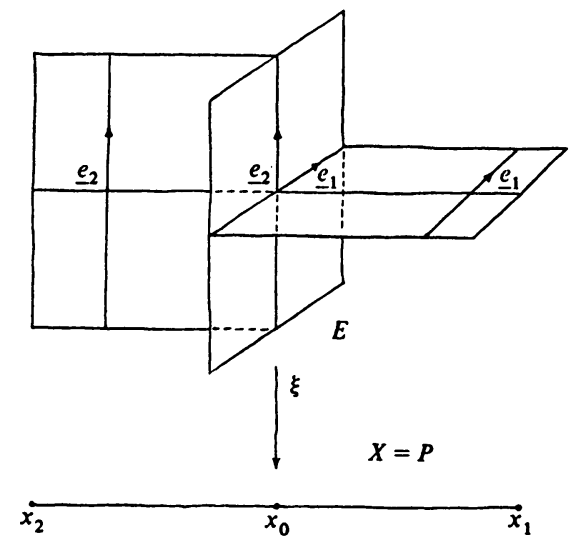

Diagram 1 
For any simplicial bundle $\xi: E \rightarrow X$, the inverse image $\xi^{-1}(x)$ of any $x \in X$ is given by the data $\left\{\phi_{\alpha}(x,-) F_{\alpha} ; l_{\beta \alpha}\right\}$ (Axiom 5 of Definition 2.1). This is why in Example 2.2 the structure fiber $F_{\alpha}=R^{0}$ of $\xi$ associated to the simplicial chart with domain $U_{\alpha}=\left\{x_{0}\right\}$ and the inverse image $\xi^{-1}\left(x_{0}\right)=R^{2}$ of $x_{0}$ are so radically different.

The following result helps motivate Definition 2.1 (see [13]). First, however, observe that a small open subset $U$ of a simplicial space $X$ may be viewed as the free union $\amalg V_{\alpha}$ of copies of open subsets $V_{\alpha} \subseteq s_{\alpha}^{n}$ ( $n$ may vary with $\alpha$ ) under the identification $R: x_{\alpha} R x_{\beta}$ iff $x_{\beta}$ is the image of $x_{\alpha}$ under $\psi_{\beta} \circ \psi_{\alpha}^{-1}$, i.e., $U=\amalg V_{\alpha} / R$.

2.3. THEOREM. There is a functor $\tau$ from the category of simplicial spaces to the category of simplicial bundles

$$
\tau: X \leadsto(\tau(X): T X \rightarrow X)
$$

which satisfies the following criteria:

(1) $\tau(V)=\pi_{1}: T V=V \times R^{n} \rightarrow V$ for every open subset $V \subseteq s^{n}$, for any $n, \pi_{1}$ denoting projection onto the first factor,

(2) if $U=\amalg V_{\alpha} / R$ is a small open subset of the simplicial space $X$ as described above, then $\tau(U)$ is the simplicial bundle generated by

$$
\pi: \amalg T V_{\alpha} / R_{*} \rightarrow U
$$

where $R_{*}$ is the identification: $v_{\alpha} R_{*} v_{\beta}$ iff $v_{\beta}$ is the image of $v_{\alpha}$ under the Jacobian $\left(\psi_{\beta} \circ \psi_{\alpha}^{-1}\right)_{*} \circ \psi_{\beta} \circ \psi_{\alpha}^{-1}$, and where $\pi$ is the map induced by the maps $\psi_{\alpha}^{-1} \circ \tau\left(V_{\alpha}\right)$, and

(3) if $U$ is a small open subset of the simplicial space $X$ then $\tau(U)$ is the restriction of $\tau(X)$ to $T U=\tau(X)^{-1} U$.

(Note. In criterion (2) above, "generated" means that, for each $x \in U$, $\tau(U)^{-1} x$ is the smallest vector space which spans $\amalg \tau\left(V_{\alpha}\right)^{-1} x / R_{*}$.)

Proof. First let us recall that if $X$ is a simplicial space and $U$ is a small open neighborhood of $x_{0} \in X$, then a coordinate system $u$ on $U$ (with origin $x_{0}$ ) is a collection of piecewise affine functions $u_{k}: U \rightarrow R$ such that, for each wedge $U_{\alpha}$ of $U, u_{k \mid U_{\alpha}}=0$ except for a subset of $\operatorname{dim} U_{\alpha}$ functions, and the set $\left\{u_{\alpha}^{*}\right\}$ of functions $u_{\alpha}^{k}=u_{k \mid U_{\alpha}}$ for $u_{k}$ in this subset forms a coordinate system on $U_{\alpha}$ (with origin $x_{0} \in U_{\alpha}$ ). If $x_{0}$ is the origin of the coordinate system $u$ on $U$ then $U$ is necessarily a small open neighborhood of $x_{0}$.

If $X$ is a simplicial space and $U$ is a small open neighborhood of $x_{0} \in X$, let $V$ be the vector space which has for a basis the vectors $\left\{\left(\partial / \partial u_{k}\right)_{x_{0}}: u_{k} \in u\right\}$. Let $T U$ be the subspace of $U \times V$ consisting of all those $(x, v)$ for which the $k$ th component $c_{k}$ of $v=\sum c_{k}\left(\partial / \partial u_{k}\right)_{x_{0}}$ is nonzero only if there is a wedge $U_{\alpha}$ 
of $U$ for which $x \in U_{\alpha}$ and $u_{k \mid U_{\alpha}} \in\left\{u_{\alpha}^{*}\right\}$, and let $\tau(U): T U \rightarrow U$ be projection onto the first factor. Then:

(1) if $U_{\alpha}$ is a wedge of $U$ and $\tilde{F}_{\alpha}$ is the vector space with basis $\left\{\left(\partial / \partial u_{\alpha}^{k}\right)_{x_{0}}: u_{\alpha}^{k} \in\left\{u_{\alpha}^{*}\right\}\right\}$, then the map $\tilde{\phi}_{\alpha}: U_{\alpha} \times \tilde{F}_{\alpha} \rightarrow T U$ given by

$$
\tilde{\phi}_{\alpha}\left(x, \Sigma c_{k}\left(\partial / \partial u_{\alpha}^{k}\right)_{x_{0}}\right)=\left(x, \Sigma c_{k}\left(\partial / \partial u_{k}\right)_{x_{0}}\right)
$$

is a fiber preserving embedding which is a linear injection on each fiber,

(2) if $U_{\alpha}$ and $U_{\beta}$ are wedges of $U$ for which $U_{\alpha} \subseteq U_{\beta}$, then $\tilde{\phi}_{\alpha}(x,-)$ $=\tilde{\phi}_{\beta}(x,-) \circ \tilde{l}_{\beta \alpha}$ for every $x \in U_{\alpha}$ where $\tilde{l}_{\beta \alpha}$ is the (Jacobian) map given on generators by $\tilde{l}_{\beta \alpha}\left(\partial / \partial u_{\alpha}^{k}\right)_{x_{0}}=\left(\partial / \partial u_{\beta}^{k}\right)_{x_{0}}$, and

(3) if $U_{\alpha}, U_{\beta}$, and $U_{\gamma}$ are wedges of $U$ for which $U_{\alpha} \subseteq U_{\beta} \subseteq U_{\gamma}$, then $\tilde{l}_{\gamma \alpha}=\tilde{l}_{\gamma \beta} \circ \tilde{l}_{\beta \alpha}$.

The global construction of $\tau$ follows from the following facts (see [11):

2.4. Fact. If $u$ is a coordinate system on the small open subset $U$ with origin $x_{0}$, then for any other $x_{1} \in U$, a coordinate system on a small open neighborhood $W \subseteq U$ of $x_{1}$ with origin $x_{1}$ may be obtained by translating coordinate functions in some subset of $u$.

2.5. Fact. If $u^{\prime}$ and $u^{\prime \prime}$ are coordinate systems on the small open subset $U$ both with origin $x_{0} \in U$, then for every $u_{j} \in u^{\prime}$ there are unique real constants $c_{j k}$ for which $u_{j}=\sum c_{j k} u_{k}, u_{k} \in u^{\prime \prime}$.

The total space $T X$ of $\tau(X)$ is defined by covering $X$ with all possible combinations of small open subsets $U$ equipped with coordinate systems $u$ and letting $T X$ be the free union of all the spaces $T U$ as constructed above modulo the identification $T:\left(x^{\prime}, v^{\prime}\right) T\left(x^{\prime \prime}, v^{\prime \prime}\right)$ iff $x^{\prime}=x^{\prime \prime}$ and, after translating coordinate systems $u^{\prime}$ and $u^{\prime \prime}$ to $x^{\prime}$ and $x^{\prime \prime}$, respectively, $v^{\prime}=\sum_{j k} c_{k} c_{j k}\left(\partial / \partial u_{j}\right)_{x}$ for $u_{j} \in u^{\prime}$ where $x=x^{\prime}=x^{\prime \prime}$, where $v^{\prime \prime}=\sum_{k} c_{k}\left(\partial / \partial u_{k}\right)_{x}, u_{k} \in u^{\prime \prime}$, and where the constants $c_{j k}$ are given by $u_{j}=\sum c_{j k} u_{k}$. Let $\tau(X): T X \rightarrow X$ be the induced projection.

To show that $\tau(X)$ is a simplicial bundle, for every simplicial chart $\left(U_{\alpha}, \psi_{\alpha}\right)$ on $X$ let $U$ be a small open subset of $X$ for which $U_{\alpha}$ is a wedge of $U$, and let $u$ be a coordinate system on $U$. Let $F_{\alpha}$ be the structure fiber $F_{\alpha}$ of $\tau(U): T U$ $\rightarrow U$ associated to $U_{\alpha}$, and let $\phi_{\alpha}: U_{\alpha} \times F_{\alpha} \rightarrow T X$ be $\tilde{\phi}_{\alpha}$ followed by inclusion. If $\left(U_{\alpha}, \psi_{\alpha}\right)$ and $\left(U_{\beta}, \psi_{\beta}\right)$ are simplicial charts on $X$ for which $U_{\alpha} \subseteq U_{\beta}$, let $l_{\beta \alpha}=\tilde{l}_{\beta \alpha}$.

Finally, if $u^{\prime}$ and $u^{\prime \prime}$ are two different coordinate systems on $U$ both with origin $x_{0}$ then there is a linear isomorphism $l: F_{\alpha}^{\prime \prime} \rightarrow F_{\alpha}^{\prime}$ for which $\phi_{\alpha}^{\prime \prime}(x,-)$ $=\phi_{\alpha}^{\prime}(x,-) \circ l$ for each $x \in U_{\alpha}: l$ is described on generators by $l\left(\partial / \partial u_{\alpha}^{k}\right)$ $=\sum_{j} c_{j k}\left(\partial / \partial u_{\alpha}^{j}\right)$. To verify that $\phi_{\alpha}^{\prime \prime}(x,-)=\phi_{\alpha}^{\prime}(x,-) \circ l$, observe that $\phi_{\alpha}^{\prime}(x$, $\left.l\left(\partial / \partial u_{\alpha}^{k}\right)\right)=\left(x, \sum_{j}^{\prime} c_{j k}\left(\partial / \partial u_{j}\right)\right)$ where the summation is taken over all $j$ for which $u_{j \mid U_{\alpha}} \in\left\{u_{\alpha}^{*}\right\}^{\prime}$, and that $\phi_{\alpha}^{\prime \prime}\left(x, \partial / \partial u_{\alpha}^{k}\right)=\left(x, \Sigma_{j} c_{j k}\left(\partial / \partial u_{j}\right)\right)$ where the 
summation is taken over all $j$. The conclusion follows since $c_{j k}=0$ if $u_{j \mid U_{\alpha}}=0$ and $u_{k \mid U_{\alpha}} \in\left\{u_{\alpha}^{*}\right\}^{\prime \prime}$. Q.E.D.

2.6. DEFINITION. The functor $\tau$ is the tangent functor, and the total space $T X$ of the tangent bundle $\tau(X): T X \rightarrow X$ of the simplicial space $X$ is the tangent space of $X$.

2.7. EXAMPLE. If $X=R^{n}$ ( $R^{n}$ being equipped with the trivial simplicial structure), then the tangent bundle of $X$ is precisely the classical smooth tangent (vector) bundle of $R^{n}$, namely $\tau(X)=\pi_{1}: T X=X \times R^{n} \rightarrow X, \pi_{1}$ denoting projection onto the first factor.

2.8. EXAMPLE. The simplicial bundle $\xi: E \rightarrow X$ constructed in Example 2.2 is the tangent object $\tau(X): T X \rightarrow X$ of the simplicial space $X=P$.

2.9. REMARK. The construction of tangent objects for simplicial spaces is natural in the sense that if $f: X_{1} \rightarrow X_{2}$ is a map of simplicial spaces, then there is a natural way to define the Jacobian $f_{*}: T X_{1} \rightarrow T X_{2}$ of $f$ and a corresponding morphism $\tau\left(X_{1}\right) \rightarrow \tau\left(X_{2}\right)$ of simplicial bundles. It is easy to show that $\mathrm{id}_{*}=$ id: $T X \rightarrow T X$ for id: $X \rightarrow X$ the identity map, and that given simplicial maps $f: X_{1} \rightarrow X_{2}$ and $g: X_{2} \rightarrow X_{3},(g \circ f)_{*}=g_{*} \circ f_{*}$.

It is Theorem 2.3 which will clarify the relation between de Rham theory and vector field theory for simplicial spaces (see Remark 2.10 and Definition 2.11), and the relation between vector fields and piecewise smooth flows on simplicial spaces (see Proposition 3.6).

2.10. ReMARK. Having introduced Riemannian metrics on cosimplicial bundles, one can associate a simplicial bundle $\xi$ to each metrizable cosimplicial bundle $\xi^{*} ; \xi$ is the dual of $\xi^{*}$. Dualization is a natural equivalence between the categories of metrizable simplicial and metrizable cosimplicial bundles. The tangent objects and cotangent objects of simplicial spaces are metrizable and correspond to each other under dualization (see \$5).

We now define sections of simplicial bundles. A section of a simplicial bundle $\xi: E \rightarrow X$ will be a function (not necessarily a continuous function) $s: X \rightarrow E$ for which $\xi \circ s=\mathrm{id}_{X}$ and which satisfies certain criteria. Sections are not required to be continuous since continuity implies excessive restrictions: in Example 2.2, for instance, a continuous function $s: X \rightarrow E$ for which $\xi \circ s=\mathrm{id}_{X}$ would necessarily be zero at $x_{0}$.

2.11. Definition. A section $s$ of the simplicial bundle $\xi: E \rightarrow X$ is a (not necessarily continuous) function $s: X \rightarrow E$ such that $\xi \circ s=\mathrm{id}_{X}$ and such that for every small open subset $U$ of $X$ there are piecewise smooth sections $s_{\alpha}$ of some of the product vector bundles $U_{\alpha} \times F_{\alpha} \rightarrow U_{\alpha}, U_{\alpha}$ a wedge of $U$ and $F_{\alpha}$ the structure space of $\xi$ associated to $U_{\alpha}$, for which:

(1) for every wedge $U_{\beta}$ of $U$ there is a wedge $U_{\alpha}$ of $U$ containing $U_{\beta}$ for which $s_{\alpha}$ is defined,

(2) if $U_{\alpha}$ and $U_{\beta}$ are wedges of $U$ for which $U_{\alpha} \subseteq U_{\beta}$ and for which sections 
$s_{\alpha}$ and $s_{\beta}$ are defined, then $s_{\beta \mid U_{\alpha}}=\left(\mathrm{id} \times l_{\beta \alpha}\right) \circ s_{\alpha}$, and

(3) for each $x \in U, s(x) \stackrel{=}{=}\left(\phi_{\alpha} \circ s_{\alpha}\right) x$, the summation taken over all $\alpha$ for which $x \in U_{\alpha}, s_{\alpha}$ is defined, and the $\left(\phi_{\alpha} \circ s_{\alpha}\right) x$ are distinct.

A section of the tangent object $\tau(X)$ of the simplicial space $X$ is a vector field on $X$.

The remainder of this paper is concerned with studying vector fields on simplicial spaces.

In [7], Hopf obtains results for "vector fields" on a class of spaces which can be embedded in Euclidean space. If, for example, $P$ is a polyhedron embedded in $R^{N}$ in general position, then a "vector field" on $P$ in Hopf's sense is an association of a tangent vector in $T_{x} R^{N}$ to each point $x \in P$ which satisfies two conditions. Such "vector fields" can naturally be constructed in either of two ways: First a "vector field" can be constructed from a small transformation $f: P \rightarrow P$ of $P$; such a map is a "vector field" in the sense that it corresponds to a right inverse $s: P \rightarrow P \times P \subseteq P \times R^{N}$ to projection $\pi_{1}$ : $P \times R^{N} \rightarrow P$ onto the first factor: $s(x)=(x, f(x))$. Second, in order to obtain results which are local (i.e., only depend on behavior in arbitrarily small neighborhoods of points), one can consider sequences $f_{k}: P \rightarrow P, k=1,2$, ..., of small transformations of $P$ which converge uniformly to the identity id: $P \rightarrow P$; the value at the point $x \in P$ of the "vector field" $s$ associated to such a sequence of small transformations is given by

$$
s(x)=\lim _{k \rightarrow \infty} \frac{\left(x, f_{k}(x)\right)}{\left|\left(x, f_{k}(x)\right)\right|},
$$

if this limit exists.

With respect to this latter construction, the point in talking about simplicial bundles is that the set of "tangent vectors" produced in this manner span a subspace of $T R^{N}$ which is intrinsic to $P$, i.e., which can be defined independently of an embedding of $P$ in $R^{N}$. In fact, if $r: P \rightarrow R^{N}$ is any embedding of $P$ in $R^{N}$ in general position, then this subspace is precisely the image $r_{*} T P$ of the tangent space TP of $P$ under the Jacobian $r_{*}$ of $r$, and the following diagram commutes:

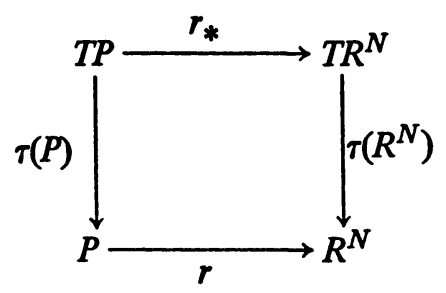

Furthermore, the constructions in [7] can be performed without embedding $P$ in $R^{N}$. 
3. Piecewise smooth flows. After defining piecewise smooth flows on simplicial spaces we will show that every piecewise smooth flow on the simplicial space $X$ generates a vector field on $X$, and conversely that every vector field on $X$ which satisfies certain integrability conditions generates a piecewise smooth flow on $X$.

3.1. Definition. A preflow $F$ on the $n$-corner $s^{n}$ is a continuous function $F: s^{n} \times R \rightarrow s^{n}$, where $\underline{s}^{n} \times R$ is a (not necessarily open) subset of $s^{n} \times R$ containing $s^{n} \times\{0\}$, such that:

(1) for each $x_{0}$ in the interior of $s^{n}$ there is a neighborhood $N \times J \subseteq \underline{s^{n} \times R}$ of $\left(x_{0}, 0\right)$ which is open in $s^{n} \times R$ and over which $F$ is a smooth flow,

(2) if $F(x, t)=\left(F_{k}(x, t)\right)$ and $\stackrel{\circ}{F}_{k}(x, t)$ is the restriction of $F_{k}(x, t)$ to the interior of $s^{n} \times R$, then $\left(\partial \dot{F}_{k}(x, t) / \partial t\right)_{\mid t=0}$ extends to a (necessarily unique) smooth function $\left(\partial F_{k}(x, t) / \partial t\right)_{\mid t=0}$ on $s^{n}$, and

(3) if $F^{\prime}:\left(s^{n} \times R\right)^{\prime} \rightarrow s^{n}$ is any map satisfying the above conditions and $F(x, t)=F^{\prime}(x, t)$ for all $(x, t)$ in $\underline{\left(s^{n} \times R\right)} \cap \underline{\left(s^{n} \times R\right)^{\prime}}$ then $\underline{s^{n} \times R}=\underline{\left(s^{n} \times R\right)^{\prime}}$.

The relative version of a preflow is a map $F: \underline{W \times I} \rightarrow s^{n}$, where $W$ is some small open subset of $s^{n}$ and $I$ is some interval neighborhood of $0 \in R$, for which the relative versions of the above criteria are met.

If $\partial / \partial x_{k}, k=1,2, \ldots$, are the standard tangent vector fields on $s^{n}$ then a preflow $F$ on the $n$-corner $s^{n}$ naturally induces the vector field

$$
\sum\left(\partial F_{k}(x, t) / \partial t\right)_{\mid t=0}\left(\partial / \partial x_{k}\right)
$$

on $s^{n}$. Preflows are introduced to establish a converse to this construction. In general the concept of flow will not suffice: On $s^{2}$, for example, consider the vector field $v=\partial / \partial x_{1}-\partial / \partial x_{2}$. If this vector field is extended to the smooth vector field $\underline{v}=\partial / \partial x_{1}-\partial / \partial x_{2}$ on $R^{2}$, then the smooth flow $\underline{F}$ on $R^{2}$ which generates $\underline{v}$ is given by $\underline{F}\left(x_{1}, x_{2}, t\right)=\left(x_{1}+t, x_{2}-t\right)$. Restricting $\underline{F}$ to

$$
\underline{s^{2} \times R}=\left\{\left(x_{1}, x_{2}, t\right) \in s^{2} \times R: F\left(x_{1}, x_{2}, t\right) \in s^{2}\right\}
$$

we obtain a preflow $F$ on $s^{2}$ which generates $v$. Observe that the only value of $t$ for which $(0,0, t)$ is in the domain of $F$ is $t=0$.

3.2. LeMMA. Let $U$ and $W$ be small open neighborhoods of the origin $0 \in s^{n}$ which have compact closure and for which the closure of $W$ is contained in $U$. If $\sum f_{k}\left(\partial / \partial x_{k}\right)$ is any vector field on $U\left(\right.$ where $f_{k} \in A(U)$ for each $\left.k\right)$ then there is a unique preflow $F: \underline{W \times I} \rightarrow U$ for which $f_{k}=\left(\partial F_{k}(x, t) / \partial t\right)_{\mid t=0}$ for each $k$.

Proof. For $c \in R$ let $c=(c+|c|) / 2 \in R^{+}$, and for $x=\left(x_{k}\right) \in R^{n}$ let $\underline{x}=\left(\underline{x}_{k}\right) \in s^{n}$. On the open neighborhood

$$
\underline{U}=\left\{x \in R^{n}: \underline{x} \in U \text { and } x_{k}>-1 \forall k\right\}
$$


of $0 \in R^{n}$ define $f_{k}: \underline{U} \rightarrow R$ by $f_{k}(x)=f_{k}(\underline{x})$, for each $k$, and $\underline{f}=\left(f_{k}\right): \underline{U}$ $\rightarrow R^{n}$; let $\underline{W}=\left\{x \in \underline{U}: \underline{x} \in W\right.$ and $\left.x_{k}>-\frac{1}{2} \forall k\right\}$. Since $\underline{U}$ and $\underline{W}$ have compact closure it is not difficult to verify the Lipschitz condition

$$
\left|f\left(x_{0}\right)-f\left(x_{1}\right)\right|<K\left|x_{0}-x_{1}\right|
$$

for $x_{0}, x_{1} \in W$ and some fixed constant $K$.

Now apply Picard's method of successive approximations (see [6]) to the equations

$$
\partial \underline{F}(x, t) / \partial t=\underline{f}(F(x, t)), \quad F(x, 0)=x,
$$

to obtain a unique flow $F: \underline{W} \times I \rightarrow \underline{U}$, and define the preflow $F$ on $W$ by restricting $F$ to

$$
\underline{W \times I}=\{(x, t) \in W \times I: F(x, t) \in U\} .
$$

The remainder of the lemma is immediate. Q.E.D.

It is not difficult to extend Lemma 3.2 to the case in which $U$ and $W$ are small open neighborhoods of any point $x_{0} \in s^{n}$.

3.3. Definition. A piecewise smooth flow $F$ on the simplicial space $X$ is a map $F: X \times I \rightarrow X, I$ an interval neighborhood of the origin $0 \in R$, such that:

(1) $F(x, 0)=x$ and $F\left(x, t_{1}+t_{2}\right)=F\left(F\left(x, t_{1}\right), t_{2}\right)$ for all $x \in X$ and $t_{1}, t_{2}$ $\in I$ for which $t_{1}+t_{2} \in I$, and

(2) if $U$ and $W$ are small open subsets of $X$ for which the closure of $W$ is contained in $U$, then for every wedge $U_{\beta}$ of $U$ there is a wedge $U_{\alpha}$ of $U$ for

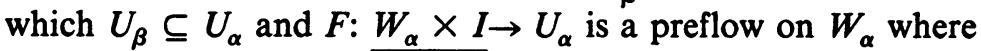

$$
\underline{W_{\alpha} \times I}=\left\{(x, t) \in W_{\alpha} \times I: F(x, t) \in U_{\alpha}\right\}
$$

and $W_{\alpha}=W \cap U_{\alpha}$.

The purpose of the following result is to show existence of piecewise smooth flows.

3.4. Proposition. Let $T: X \rightarrow R^{n}$ be a triangulation of $R^{n}$ by a simplicial space $X$. If $F: R^{n} \times R \rightarrow R^{n}$ is a smooth flow on $R^{n}$, then the map

$$
T^{-1} \circ F \circ(T \times \text { id }): X \times R \rightarrow X
$$

is a piecewise smooth flow on $X$.

Proof. The first condition of Definition 3.3 is immediate. To verify the second condition, let $U$ and $W$ be small open subsets of $X$ as indicated. Then for every wedge $U_{\beta}$ of $U$ there is a wedge $U_{\alpha}$ of $U$ of dimension $n$ containing $U_{\beta}$, and it is easy to show that $F: W_{\alpha} \times R \rightarrow U_{\alpha}$ is a preflow. Q.E.D.

3.5. EXAMPLE. Consider the triangulation $T: X \rightarrow R^{1}$ of $R^{1}$ illustrated in Diagram 2 which is induced by the identity id: $R^{1} \rightarrow R^{1}$. Equip $X$ and $R^{1}$ with 
coordinate systems as illustrated. If $F: R^{1} \times R \rightarrow R^{1}$ is the smooth flow given by $F(x, t)=x+t$, then $T^{-1} \circ F \circ(T \times$ id $): X \times R \rightarrow X$ is a piecewise smooth flow on $X$. In this case

$$
F_{\mid \underline{U_{\alpha} \times R}}\left(u_{\alpha}, t\right)=u_{\alpha}+t \text { where } \underline{U_{\alpha} \times R}=\left\{\left(u_{\alpha}, t\right) \in U_{\alpha} \times R: u_{\alpha}+t \geqslant 0\right\},
$$

and

$$
F_{\mid U_{\beta} \times R}\left(u_{\beta}, t\right)=u_{\beta}+t \text { where } \underline{U_{\beta} \times R}=\left\{\left(u_{\beta}, t\right) \in U_{\beta} \times R: u_{\beta}-t \geqslant 0\right\} .
$$

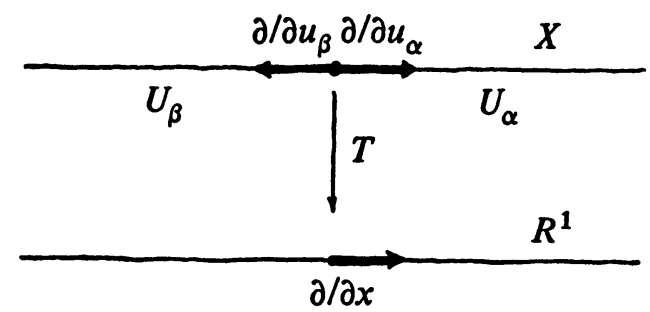

Diagram 2

The next result relates piecewise smooth flows and vector fields on simplicial spaces.

Let $U$ and $W$ be small open subsets of the simplicial space $X$ which have compact closure and for which the closure of $W$ is contained in $U$, and for every wedge $U_{\alpha}$ of $U$ let $W_{\alpha}=W \cap U_{\alpha}$.

3.6. Proposition. If $F: W \times I \rightarrow U$ is a piecewise smooth flow on $W$ then:

(1) for every wedge $U_{\beta}$ of $U$ there is a wedge $U_{\alpha}$ of $U$ containing $U_{\beta}$ for which

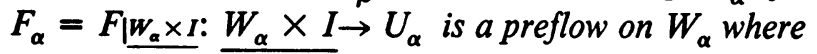

$$
\underline{W_{\alpha} \times I}=\left\{(x, t) \in W_{\alpha} \times I: F(x, t) \in U_{\alpha}\right\},
$$

and

(2) if $U_{\alpha}$ and $U_{\beta}$ are wedges of $U$ for which $U_{\alpha} \subseteq U_{\beta}$ and for which preflows $F_{\alpha}$ and $F_{\beta}$ are defined, then $F_{\beta \mid W_{\alpha} \times I}=F_{\alpha}$.

Conversely, a collection of preflows $F_{\alpha}: \underline{W_{\alpha} \times R} \rightarrow U_{\alpha}$ defined on some of the wedges $W_{\alpha}$ of $W$ such that:

(3) there is a positive number $\varepsilon$ such that, for each $(x, t) \in W \times(-\varepsilon, \varepsilon),(x, t)$ $\in W_{\alpha} \times R$ for some wedge $W_{\alpha}$ of $W\left(W_{\alpha}\right.$ may vary with $\left.t\right)$, and

(4) whenever $(x, t) \in\left(W_{\alpha} \times R\right) \cap\left(W_{\beta} \times R\right), F_{\alpha}(x, t)=F_{\beta}(x, t)$, generates a piecewise smooth flow $F: W \times I \rightarrow U, I$ an interval neighborhood of $0 \in R$. Furthermore, if $F: W \times I \rightarrow U$ is a piecewise smooth flow and the corresponding collection of preflows $F_{\alpha}: W_{\alpha} \times I \rightarrow U_{\alpha}$ satisfies conditions (3) and (4), then the piecewise smooth flow constructed from the $F_{\alpha}$ 's is $F$ : and if $F$ 
is a piecewise smooth flow constructed from a collection of preflows satisfying conditions (3) and (4) then the collection of preflows generated by $F$ contains each original preflow.

Proof. The first part of this result is immediate.

Given a collection of preflows $F_{\alpha}: \underline{W_{\alpha} \times R} \rightarrow U_{\alpha}$ defined on some of the wedges $W_{\alpha}$ of $W$ which satisfy conditions (3) and (4), let $I$ denote the largest possible open interval $(-\varepsilon, \varepsilon)$ for which condition (3) holds. For $(x, t) \in W \times I$ we define $F(x, t)$ as $F_{\alpha}(x, t)$ for any $\alpha$ for which $(x, t) \in W_{\alpha} \times R$; this $\alpha$ exists by condition (3), and $F(x, t)$ is well defined by condition (4).

The remainder of this result is immediate. Q.E.D.

3.7. Corollary. Every piecewise smooth flow $F: X \times I \rightarrow X$ on the simplicial space $X$ generates a vector field on $X$. Conversely let $s: X \rightarrow T X$ be a vector field on the compact simplicial space $X$. If, for all choices of $U$ and $W$ as above, the piecewise smooth sections $s_{\alpha}: U_{\alpha} \rightarrow T U_{\alpha}=U_{\alpha} \times F_{\alpha}$ defined on the wedges $U_{\alpha}$ of $U$ generate preflows $F_{\alpha}: W_{\alpha} \times R \rightarrow U_{\alpha}$ on $W_{\alpha}$ which satisfy conditions (3) and (4) of Proposition 3.6, then s generates a piecewise smooth flow on $X$.

Proof. If $U$ and $W$ are small open neighborhoods of $x \in U$ for which the closure of $W$ is contained in $U$, then, with the notation of Proposition 3.6, each preflow $F_{\alpha}: W_{\alpha} \times I \rightarrow U_{\alpha}$ generates a vector field $s_{\alpha}: W_{\alpha} \rightarrow T W_{\alpha}$ on $W_{\alpha}$. We define $s: X \rightarrow T X$ by $s(x)=\Sigma\left(\phi_{\alpha} \circ s_{\alpha}\right) x$, the summation taken over all $\alpha$ for which $x \in U_{\alpha}, s_{\alpha}$ is defined, and the $\left(\phi_{\alpha} \circ s_{\alpha}\right) x$ are distinct. The remainder of the corollary follows immediately from Lemma 3.2. Q.E.D.

Conditions (3) and (4) correspond closely to the two conditions in [7] mentioned at the end of $\$ 2$ which are necessary for a map $s: P \rightarrow P \times R^{N}$ to be a "vector field".

We will now use Corollary 3.7 to construct a piecewise smooth flow on an arbitrary polyhedron. In this construction, and frequently throughout the sequel, we will assume the polyhedron $P$ is described by cells $B^{n}=[-1,1]^{n}$ $\subseteq R^{n}$ instead of by simplices

$$
\Delta^{n}=\left\{x=\left(x_{k}\right) \in R^{n}: x_{k} \in[0,1] \forall k \text { and } \sum x_{k}=1\right\} \subseteq R^{n} .
$$

This is done strictly for mechanical convenience: All pertinent results are valid for simplicial polyhedra as well as for cell polyhedra.

To be precise: An $n$-cell $B^{n}$ in $R^{N}$ is the image of the standard $n$-cell $B^{n}=[-1,1]^{n} \subseteq R^{n}$ under an injective linear transformation $T: R^{n} \rightarrow R^{N}$. A cell polyhedron $P$ is a collection of cells $\left\{B_{\alpha}^{n}\right\}$ in $R^{N}$, for some $N$, such that:

(1) if $B_{\alpha}^{n}$ is in $P$ then each (closed) face of $B_{\alpha}^{n}$ is in $P$, and

(2) if $B_{\alpha}^{m}$ and $B_{\beta}^{n}$ are in $P$, then $B_{\alpha}^{m} \cap B_{\beta}^{n}$ is a (unique) face of both $B_{\alpha}^{m}$ and $B_{\beta}^{n}$.

Every cell polyhedron may be topologized as a subset of Euclidean space, 
and with this topology is combinatorially equivalent to a simplicial polyhedron.

3.8. CoRollary. Every compact (or locally finite) cell polyhedron $P$ admits a nontrivial piecewise smooth flow.

Proof. Let $\phi:[-1,1] \rightarrow[0,1]$ be a smooth function for which $\phi(t)=-t^{2}$ +1 for $t \in[0,1 / 3), \phi(t)=(t-1)^{2}$ for $t \in(2 / 3,1], \phi(t)=\phi(-t)$ for $t$ $\in[-1,1]$, and $\phi^{\prime}(t)=0$ only for $t=0, \pm 1$. Define the (piecewise) smooth real valued function $f^{n}$ on the $n$-cell $B^{n}=[1,1]^{n}$ by $f^{n}(x)=\sum_{k=1}^{n} \phi\left(x_{k}\right)$.

Since $\phi( \pm 1)=0$, the functions $f_{\alpha}^{n}: B_{\alpha}^{n} \rightarrow R$ defined on the $n$-cells $B_{\alpha}^{n}$ of $P$ define a global piecewise smooth function $f: P \rightarrow R$. Observe that if $\left(U_{\alpha}, \psi_{\alpha}\right)$ is a simplicial chart on $P$ then $U_{\alpha} \subseteq B_{\alpha}^{n}$ for some $n$-cell $B_{\alpha}^{n}$, and thus $f_{\mid U_{\alpha}}=f_{\alpha \mid U_{\alpha}}^{n}$.

For every simplicial chart $\left(U_{\alpha}, \psi_{\alpha}\right)$ on $P$ let $s_{\alpha}: U_{\alpha} \rightarrow T U_{\alpha}=U_{\alpha} \times F_{\alpha}$ be the vector field given by $s_{\alpha}=\operatorname{grad} f_{\alpha}^{n}$ where $U_{\alpha} \subseteq B_{\alpha}^{n}$ (and grad = gradient). If $\left(U_{\alpha}, \psi_{\alpha}\right)$ and $\left(U_{\beta}, \psi_{\beta}\right)$ are simplicial charts on $P$ for which $U_{\alpha} \subseteq U_{\beta}$, then it is easy to verify that $s_{\beta \mid U_{\alpha}}=s_{\alpha}$. Define the continuous vector field (i.e., map) $s: P \rightarrow T P$ by letting $s(x)=s_{\alpha}(x)$ for $\left(U_{\alpha}, \psi_{\alpha}\right)$ any simplicial chart for which $x \in U_{\alpha}$ and $s_{\alpha}=\operatorname{grad} f_{\alpha}^{n}$. It is not difficult to verify conditions (3) and (4) of Proposition 3.6, and hence the conclusion follows. Q.E.D.

For example, the graphs of $f^{1}: B^{1} \rightarrow R$ and $f^{2}: B^{2} \rightarrow R$ are illustrated in Diagram 3.
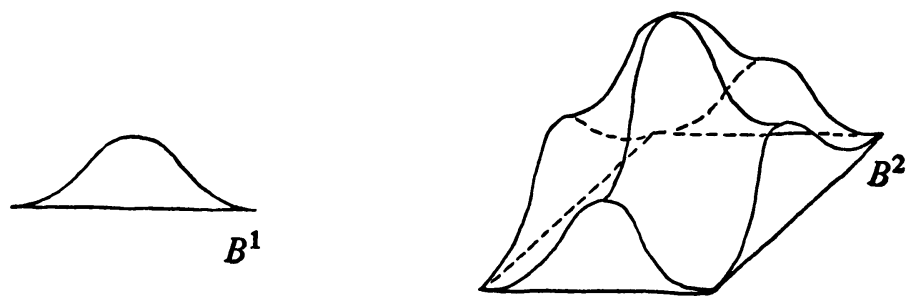

\section{DiAgRAM 3}

The flow lines for the corresponding piecewise smooth flows are illustrated in Diagram 4.
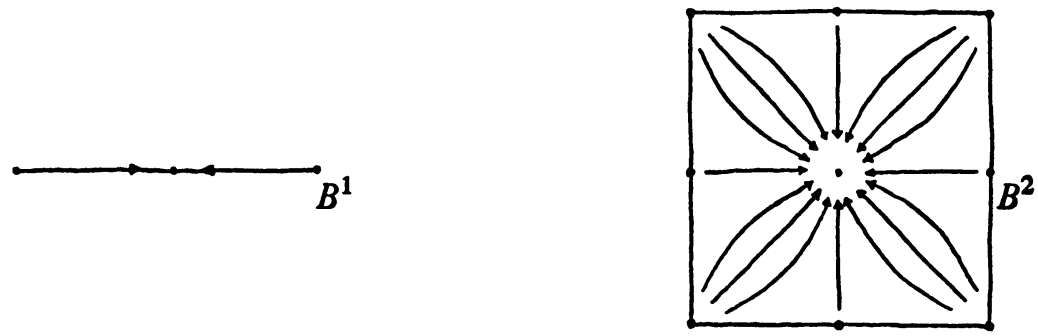

Diagram 4 
3.9. Remark. Let $x_{0}$ be the barycenter of the $m$-face $B^{m}$ of the $n$-cell $B^{n}=[-1,1]^{n}$. Let $u$ be the coordinate system obtained by translating the standard coordinate functions on $R^{n}$ to $x_{0}$, and order $u=\left\{u_{*}\right\}$ so that $\left\{u_{1}, \ldots, u_{m}\right\}$ forms a coordinate system on $B^{m}$. Then in a neighborhood of $x_{0}$,

$$
f^{n}(x)=\sum_{k=m+1}^{n} u_{k}^{2}-\sum_{k=1}^{m} u_{k}^{2}
$$

so that

$$
\operatorname{grad} f^{n}(x)=\sum_{k=m+1}^{n} 2 u_{k} \frac{\partial}{\partial u_{k}}-\sum_{k=1}^{m} 2 u_{k} \frac{\partial}{\partial u_{k}}
$$

and $F(x, t)=\left(u_{k} \exp c_{k} t\right)$ where $c_{k}=-2$ if $k=1, \ldots, m$, and $c_{k}=2$ if $k=m+1, \ldots, n$.

4. A Hopf theorem. In this section we will associate indices to fixed points of piecewise smooth flows. We will show that if $F$ is a piecewise smooth flow on the cell polyhedron $P$ which has only a finite set of fixed points then the sum of the indices of these fixed points is the Euler characteristic of $P$. Finally we will show that if $P=M$ is a closed polyhedral manifold whose Euler characteristic is zero then there is a fixed point free piecewise smooth flow on $P$.

There is an index (see [3]) which associates to every self-map $f: P \rightarrow P$ of the cell polyhedron $P$, and small open subset $U$ of $P$ on whose boundary there are no fixed points of $f$, an integer $i(f, U)$ as follows: Suppose $r$ is a realization of $P$ in $R^{N}=S^{N}-\{(0, \ldots, 0,1)\}$. Let $\rho: W \rightarrow r P$ be a retraction of an open subset $W \subseteq R^{N}$ onto $r P$. Let $V=\rho^{-1}(r U)$ and Fix $r U$ denote the set of fixed points of $f$ in $r U$ (by abuse of notation we write $f$ for $r f r^{-1}$ ). Define $d: V \rightarrow R^{N}$ by $d(x)=x-f \rho(x)$. Since $\rho$ is a retraction, $d(x)=0$ implies that $x=f(x)$ so that $d(V-$ Fix $r U) \subseteq R^{N}-\{0\}$. The inclusion $j$ of $(V, V-$ Fix $r U)$ into $\left(S^{N}, S^{N}-\right.$ Fix $\left.r U\right)$ induces an excision isomorphism. The index $i(f, U)$ is the degree of the map

$$
k^{*} \circ\left(j^{*}\right)^{-1} \circ d^{*}: H^{N}\left(R^{N}, R^{N}-\{0\}\right) \rightarrow H^{N}\left(S^{N}\right)
$$

where the map $k^{*}$ from $H^{N}\left(S^{N}, S^{N}-\operatorname{Fix} r U\right)$ to $H^{N}\left(S^{N}\right)$ is the connecting map from the exact sequence of the pair $\left(S^{N}, S^{N}-\right.$ Fix $\left.r U\right)$. A pair such as $(f, U)$ is an admissible pair.

This index is independent of realization and retraction, and furthermore satisfies the following axioms:

Axiom 1 (Localization). If $(f, U)$ is admissible and $g: P \rightarrow P$ is a map for which $g=f$ on the closure of $U$ then $i(f, U)=i(g, U)$.

Axioм 2 (номотору). If, for a homotopy $f_{t}: P \times[0,1] \rightarrow P,\left(f_{t}, U\right)$ is 
admissible for each $t \in[0,1]$ then $i\left(f_{0}, U\right)=i\left(f_{1}, U\right)$.

Axiom 3 (ADDITIVITY). If $(f, U)$ is admissible and $U_{1}, \ldots, U_{m}$ is a set of mutually disjoint small open subsets of $U$ for which $f(x) \neq x$ for $x \in U$ - $\cup_{k=1}^{m} U_{k}$, then $i(f, U)=\sum_{k=1}^{m} i\left(f, U_{k}\right)$.

Axiom 4 (NORMALIZATION). If $Q$ is a cover of $P$ by small open sets for which $\left(f, U_{k}\right)$ is admissible for all $U_{k} \in \mathcal{Q}$ and for which there are no fixed points of $f$ in the overlap of sets of $\mathcal{Q}$ then $\sum_{U_{k} \in \mathcal{Q}} i\left(f, U_{k}\right)$ is the Lefshetz number of $f$.

4.1. Definition. Let $F: X \times I \rightarrow X$ be a piecewise smooth flow on the simplicial space $X$. The point $x_{0} \in X$ is a fixed point of $F$ iff $F\left(x_{0}, t\right)=x_{0}$ for all $t \in I ; x_{0}$ is an ordinary point of $F$ iff $F\left(x_{0},-\right): I \rightarrow X$ is injective. If $x_{0}$ is a fixed point of $F$ which has a neighborhood $U$ in which there are no nonordinary points of $F$ other than $x_{0}$, then the index $i\left(F, x_{0}\right)$ of $F$ at $x_{0}$ is $i\left(F\left(-, t_{0}\right), U\right)$ for any positive $t_{0} \in R$.

This index is independent of the particular open neighborhood $U$ as a result of the additivity axiom. It is also independent of the particular positive $t_{0} \in R$ chosen: Given any other positive $t_{1} \in R, F\left(-, s t_{1}+(1-s) t_{0}\right)$ for $s \in[0,1]$ is a homotopy between $F\left(-, t_{0}\right)$ and $F\left(-, t_{1}\right)$ to which the homotopy axiom applies.

The following lemma normalizes this index in another way (i.e., other than Axiom 4). First, however, observe that a piecewise smooth flow $F: X \times I \rightarrow X$ on the simplicial space $X=R^{n}$ ( $R^{n}$ being equipped with the trivial simplicial structure) is a smooth flow on $R^{n}$. Also recall that if $s: R^{n} \rightarrow T R^{n}$ is a smooth vector field on $R^{n}$ which has an isolated zero at $x_{0}$, then the standard index $i\left(s, x_{0}\right)$ of $s$ at $x_{0}$ is defined as follows:

Introduce coordinates $x_{1}, \ldots, x_{n}$ about $x_{0}$ with origin $x_{0}$ so that $s$ $=\sum_{k=1}^{n} f_{k}\left(\partial / \partial x_{k}\right)$ where each $f_{k}: R^{n} \rightarrow R$ is smooth. Let $S^{n}(r)$ be a sphere of radius $r$ centered at $x_{0}$, and map $S^{n}(r)$ to itself by

$$
x \rightarrow r\left(\sum_{k=1}^{n} f_{k}(x)^{2}\right)^{-1 / 2}\left(f_{1}(x), \ldots, f_{n}(x)\right) .
$$

The index $i\left(s, x_{0}\right)$ is the degree of this map. If the determinant $\left|\partial f_{j} / \partial x_{k}\right|$ is nonzero at $x_{0}$ then $x_{0}$ is a nondegenerate zero of $s$, and $i\left(s, x_{0}\right)$ is plus or minus one as the determinant $\left|\partial f_{j} / \partial x_{k}\right|$ is positive or negative at $x_{0}$.

4.2. Lemma. Let $F: X \times R \rightarrow X$ be a piecewise smooth flow on the simplicial space $X=R^{n}$ and let $x_{0}$ be a fixed point of $F$ for which there are no nonordinary points of $F$ in a neighborhood $U$ of $x_{0}$ other than $x_{0}$. If $s: R^{n} \rightarrow T R^{n}$ is the smooth vector field on $R^{n}$ generated by $F$ then $i\left(F, x_{0}\right)=i\left(s, x_{0}\right)$.

Proof. Assume that $x_{0}=0 \in R^{n}$. Let $W \subseteq U$ be a neighborhood of $x_{0}$ and $I$ an interval neighborhood of $0 \in R$ for which $F: W \times I \rightarrow U$. Pick a positive $t_{0} \in I$ and let $f: W \rightarrow U$ be given by $f(-)=F\left(-, t_{0}\right)$. Letting $\rho: W$ 
$\rightarrow W$ be the retraction given by the identity we have $x-f \rho(x)=x-f(x)$ $=F(x, 0)-F\left(x, t_{0}\right)$; using the homotopy $W \times[0,1] \rightarrow U$ given by

$$
(x, s) \rightarrow\left(F(x, 0)-F\left(x, s t_{0}\right)\right) / s
$$

we may conclude that $x-f \rho(x)$ is homotopic to $(\partial F(x, t) / \partial t)_{\mid t=0}$ on $W$, and hence on $W-\{0\}$. Since $(\partial F(x, t) / \partial t)_{\mid t=0}$ has a zero only at the origin $0 \in R^{n}, x-f \rho(x)$ is homotopic to

$$
(\partial F(x, t) / \partial t)_{\mid t=0} /\left|(\partial F(x, t) / \partial t)_{\mid t=0}\right|
$$

on $W-\{0\}$. Q.E.D.

4.3. THEOREM (HOPF). If $F: P \times R \rightarrow P$ is a piecewise smooth flow on the cell polyhedron $P$ which is ordinary except for a finite set Fix $F$ of fixed points then $\Sigma_{x_{k} \in \mathrm{Fix} F} i\left(F, x_{k}\right)$ is the Euler characteristic $\chi(P)$ of $P$.

Proof. As a result of the index axioms, if $f: P \rightarrow P$ is a map which is homotopic to the identity id: $P \rightarrow P$, and if $Q$ is a cover of $P$ by small open subsets $U_{k}$ for which $\left(f, U_{k}\right)$ is admissible for each $k$ and for which there are no fixed points of $f$ in the overlap of sets of $\mathcal{U}_{\text {, then }} \Sigma_{U_{k} \in \mathcal{Q}} i\left(f, U_{k}\right)=\chi(P)$. The result follows since, for a fixed positive $t_{0} \in R, F\left(-, s t_{0}\right)$ for $s \in[0,1]$ is a homotopy between $f(-)=F\left(-, t_{0}\right)$ and the identity id: $P \rightarrow P$. Q.E.D.

In the next example we consider a special case of the Hopf theorem. First, however, we prove a preliminary result.

4.4. LEMMA. If $F: P \times R \rightarrow P$ is the piecewise smooth flow on the compact cell polyhedron $P$ constructed in Corollary 3.8 then the barycenters of $P$ are the fixed points of $F$ and, for every barycenter $x_{0}$ of $P, i\left(F, x_{0}\right)=(-1)^{p}$ where $p$ is the dimension of the cell of $P$ whose barycenter is $x_{0}$.

Proof. The first part of the lemma is obvious.

To prove the second part, let $N$ be the cardinality of any coordinate system on a small open neighborhood of $x_{0}$ with origin $x_{0}$. Take the $p$-cell $B^{p}$ of $P$ whose barycenter is $x_{0}$ linearly to

$$
\begin{aligned}
B_{0}^{p}=\left\{x=\left(x_{k}\right) \in R^{N}: x_{k}\right. & \in[-1,1] \text { for } k=1, \ldots, p \\
\text { and } x_{k}=0 \text { for } k & =p+1, \ldots, N\},
\end{aligned}
$$

thus taking $x_{0}$ to $0 \in R^{N}$. Take each $(p+1)$-cell $B_{\alpha}^{p+1}$ in $P$ which contains $B^{p}$ linearly to a cell in $R^{N}$ whose $p$-face corresponding to $B^{p}$ coincides with $B p$, and whose opposite $p$-side lies on the cylinder

$$
\left\{x=\left(x_{k}\right) \in R^{N}: x_{k} \in[-1,1] \text { for } k=1, \ldots, p \text { and } \sum_{k=p+1}^{N} x_{k}^{2}=1\right\} ;
$$

we may assume that the images of the $B_{\alpha}^{p+1}$ lie in distinct coordinate planes 
of $R^{N}$. Extend this map to a realization $r$ of the closed star St $x_{0}$ of $x_{0}$ in $R^{m}$ by using linearity on each cell.

Let $U=D^{N}(1 / 3) \subseteq R^{N}$ be the open $N$-ball of radius $1 / 3$ centered at the origin. For some neighborhood $W \subseteq U$ of $r\left(x_{0}\right)=0 \in R^{N}$ and some interval neighborhood $I$ of $0 \in R$, define the smooth flow $F: W \times I \rightarrow U$ by

$$
F(x, t)=\left(x_{1} \exp c_{1} t, \ldots, x_{N} \exp c_{N} t\right)
$$

where $c_{k}=-2$ if $k=1, \ldots, p$, and $c_{k}=2$ if $k=p+1, \ldots, N$. Clearly

$$
E_{\mid\left(W \cap r\left(\mathrm{St} x_{0}\right)\right) \times I}=r \circ F \circ\left(r^{-1} \times \mathrm{id}\right)_{\mid\left(W \cap r\left(\mathrm{St} x_{0}\right)\right) \times I}
$$

(see Remark 3.9).

For a positive $t_{0} \in I$ define $f: W \cap r\left(\operatorname{St} x_{0}\right) \rightarrow U \cap r\left(\operatorname{St} x_{0}\right)$ by $f(-)$ $=\left(r \circ F \circ\left(r^{-1} \times \mathrm{id}\right)\right)\left(-, t_{0}\right)$ and $\underline{f}: W \rightarrow U$ by $f(-)=\underline{F}\left(-, t_{0}\right)$. It is not difficult to construct a retraction $\rho: W \rightarrow W \cap r\left(\operatorname{St} x_{0}\right)$ for which $x-f \rho(x)$ is homotopic to $x-f x$ (such a $\rho$ may be defined by collapsing the wedges of $W$ not belonging to $r\left(\operatorname{St} x_{0}\right)$ down to $\left.W \cap r\left(\operatorname{St} x_{0}\right)\right)$. Using the homotopy $W \times[0,1] \rightarrow U$ given by

$$
(x, s) \rightarrow\left(F(x, 0)-\underline{F}\left(x, s t_{0}\right)\right) / s
$$

we may conclude that $x-f \rho(x)$ is homotopic to $(\partial \underline{F}(x, t) / \partial t)_{\mid t=0}$ on $W$, hence on $W-\{0\}$. Since $(\partial \underline{F}(x, t) / \partial t)_{\mid t=0}$ has a zero only at the origin $0 \in R^{N}, x-f \rho(x)$ is homotopic to

$$
\frac{(\partial \underline{F}(x, t) / \partial t)_{\mid t=0}}{\left|(\partial \underline{F}(x, t) / \partial t)_{\mid t=0}\right|}
$$

on $W-\{0\}$. Q.E.D.

4.5. EXAMPLE. If $F: P \times R \rightarrow P$ is the piecewise smooth flow on the compact cell polyhedron $P$ constructed in Corollary 3.8 , then using Lemma 4.4, $\Sigma_{x_{k} \in \mathrm{Fix} F} i\left(F, x_{k}\right)$ is the number of even-dimensional cells of $P$ minus the number of odd-dimensional cells of $P$; this is precisely the Euler characteristic of $P$. This is the calculation described in the Introduction which is similar to a calculation of Whitney (see [14]) giving the Euler characteristic of a smooth manifold using the singularities of vector fields.

4.6. Definition. A polyhedral m-manifold $M$ is a cell polyhedron for which the closed star of each point can be mapped homeomorphically into $R^{m}$ so that the image of each cell of $M$ is a cell in $R^{m}$.

For related definitions see [4].

4.7. COROLlARY (TO LEMMA 4.4). If $M$ is a closed polyhedral m-manifold then there is a piecewise smooth flow on $M$ which is ordinary except for a finite number 
of fixed points which are on the interior of $m$-cells of $M$.

Proof. Let $x_{0}$ be a barycenter of $M$ and $r$ any realization of the closed star of $x_{0}$ in $R^{m}$. Without loss of generality we may assume that the image of the $p$-cell $B^{p}$ of $M$ of which $x_{0}$ is the barycenter under $r$ is $\left\{x=\left(x_{k}\right) \in R^{m}: x_{k} \in[-1,1]\right.$ for $k=1, \ldots, p$ and $x_{k}=0$ for all other $\left.k\right\}$. There is now a decomposition of $R^{m}$ into wedges $W_{\alpha}^{n}$ where

$$
W_{\alpha}^{n}=\left\{t x \in R^{m}: t \in R^{+} \text {and } x \in r\left(B_{\alpha}^{n}\right)\right\}
$$

for $B_{\alpha}^{n}$ an $n$-cell of $M$ containing $B^{p}$; i.e.,

(1) $R^{m}=\cup W_{\alpha}^{n}$, and

(2) $W_{\alpha}^{m}$ is an open subset of $W_{\beta}^{n}$ iff $m=n$ and $\alpha=\beta$, and

(3) for each $W_{\alpha}^{m}$ and $W_{\beta}^{n}, W_{\alpha}^{m} \cap W_{\beta}^{n}$ is another wedge in the decomposition of $R^{m}$.

Let $g: R^{m} \rightarrow R^{m}$ be an invertible map such that:

(1) $g\left(W_{\alpha}^{n}\right)=W_{\alpha}^{n}$ for each wedge $W_{\alpha}^{n}$ in the decomposition of $R^{m}$,

(2) the restriction of $g$ to each $W_{\alpha}^{n}$ is linear and $g: W_{0}^{p} \rightarrow W_{0}^{p}$ is the identity, and

(3) for each $(p+1)$-cell $B_{\alpha}^{p+1}$ of $M$ containing $B^{p}$,

$$
\begin{aligned}
& \quad g r B_{\alpha}^{p} \subseteq\left\{x=\left(x_{k}\right) \in R^{m}: x_{k} \in[-1,1]\right. \text { for } \\
& \left.\qquad k=1, \ldots, p \text { and } \sum_{k=p+1}^{m} x_{k}^{2}=1\right\}
\end{aligned}
$$

where $B_{\alpha}^{p}$ is the $p$-face of $B_{\alpha}^{p+1}$ opposite $B^{p}$.

Finally we let $r_{0}$ denote the realization of the closed star of $x_{0}$ in $R^{m}$ given by $r_{0}=g \circ r$.

The piecewise smooth flow $F: M \times R \rightarrow M$ on $M$ constructed in Corollary 3.8 is given on $r_{0}^{-1}(U)=r_{0}^{-1}\left(D^{m}(1 / 3)\right)$ by pulling back the smooth flow on $U=D^{m}(1 / 3) \subseteq R^{m}$ associated to the gradient grad $f$ of

$$
f(x)=\sum_{k=p+1}^{m} x_{k}^{2}-\sum_{k=1}^{p} x_{k}^{2}
$$

Let $x_{1} \in U$ be any point which does not lie on the image of the $(m-1)$ skeleton of $M$ under $r_{0}$. Let $T: U \rightarrow U$ be an invertible smooth transformation which takes $x_{1}$ to the origin $0 \in R^{m}$ and which is the identity on $U-U_{0}$ where $U_{0}$ is an open neighborhood of the origin $0 \in R^{m}$ containing $x_{1}$ and whose closure is contained in $U$. Then $f \circ T: U \rightarrow R$ is a smooth function which has a critical point at $x_{1}$ and no other critical points in $U$. Thus $f \circ T$ generates a smooth flow $F$ on $U$ which has a fixed point at $x_{1}$ and which agrees 
with the flow induced by $f$ outside $U_{0}$. Pulling this flow back to $M$ we obtain a piecewise smooth flow $F^{\prime}$ on $M$ which agrees with $F$ on $M-r_{0}^{-1}\left(U_{0}\right)$ and which has a fixed point only at $r_{0}^{-1}\left(x_{1}\right)$, a point which does not lie on the $(m-1)$-skeleton of $M$. Performing this construction at each fixed point of $F$, i.e., at each barycenter of $M$, we obtain the desired result. Q.E.D.

4.8. THEOREM (HOPF). If $M$ is a closed polyhedral m-manifold whose Euler characteristic is zero, then there is a piecewise smooth flow on $M$ which is ordinary at every point.

Proof. Let $F: M \times I \rightarrow M$ be the piecewise smooth flow on $M$ constructed in Corollary 4.7. Order the $m$-cells of $M: B_{0}^{m}, \ldots, B_{N}^{m}$ so that each $B_{k}^{m}$ has an $(m-1)$-face in common with a previous $B_{i}^{m}$; for each $k$ pick one such $(m-1)$-cell $B_{k}^{m-1}$ and let $x_{k}$ be the barycenter of $B_{k}^{m-1}$.

For each $k=1,2, \ldots, N$ there are realizations $r_{k}$ : St $x_{k} \rightarrow V_{k} \subseteq R^{m}$ and small open neighborhoods $U_{k}$ and $W_{k}$ of $x_{k}$ for which the closure of $W_{k}$ is contained in $U_{k}$ and such that

$$
r_{k} \circ F \circ\left(r_{k}^{-1} \times \mathrm{id}\right): r_{k}\left(W_{k}\right) \times I_{k}^{\prime} \rightarrow r_{k}\left(U_{k}\right)
$$

is a smooth flow for some interval neighborhood $I_{k}^{\prime}$ of $0 \in R$. For $k=0,1$, $\ldots, N$, let $\underline{U}_{k}$ and $\underline{W}_{k}$ be open subsets of the interior of $B_{k}^{m}$ for which the closure of $\underline{W}_{k}$ is contained in $\underline{U}_{k}$, and:

(1) all the fixed points of $F$ in $B_{k}^{m}$ lie in $\underline{W}_{k}$ for $k=0,1, \ldots, N$,

(2) $\underline{W}_{k} \cap W_{k} \neq \varnothing$ and $\underline{W}_{k-1} \cap W_{k} \neq \varnothing$ for $k=1, \ldots, N$, and

(3) there is an interval neighborhood $I_{k}^{\prime \prime}$ of $0 \in R$ for which $F: \underline{W}_{k} \times I_{k}^{\prime \prime}$ $\rightarrow \underline{U}_{k}$.

(See Diagram 5.)
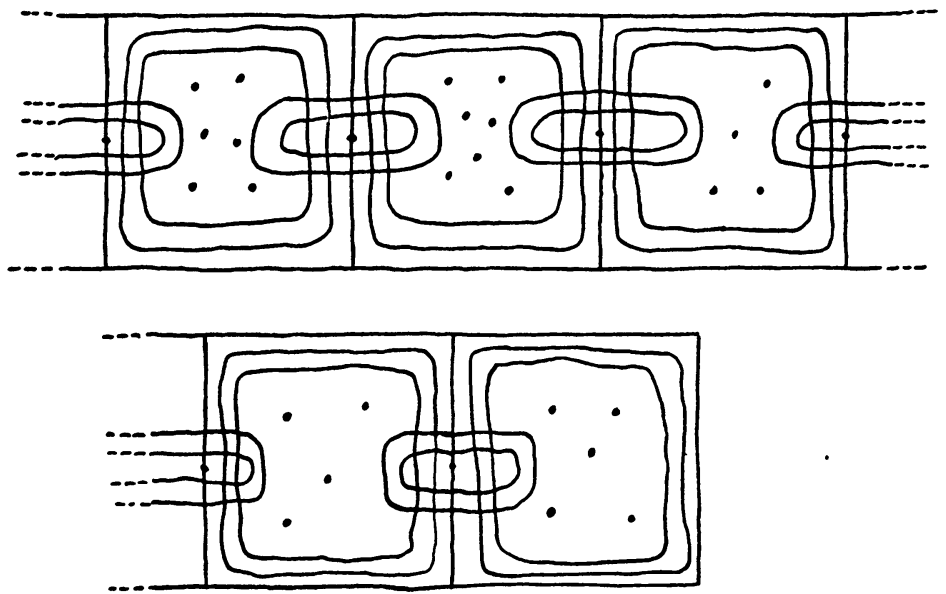

Diagram 5 
If $I_{N}=I_{N}^{\prime} \cap I_{N}^{\prime \prime}$, then

$$
r_{N} \circ F \circ\left(r_{N}^{-1} \times \mathrm{id}\right): r_{N}\left(\underline{W}_{N} \cup W_{N}\right) \times I_{N} \rightarrow r_{N}\left(\underline{U}_{N} \cup U_{N}\right)
$$

is a smooth flow which has a finite number of fixed points which are in the interior of $r_{N}\left(\underline{W}_{N} \cup W_{N}\right)$. This flow may be smoothly altered to a smooth flow

$$
r_{N} \circ F_{N} \circ\left(r_{N}^{-1} \times \mathrm{id}\right): r_{N}\left(\underline{W}_{N} \cup W_{N}\right) \times I_{N} \rightarrow r_{N}\left(\underline{U}_{N} \cup U_{N}\right)
$$

which agrees with $r_{N} \circ F \circ\left(r_{N}^{-1} \times\right.$ id $)$ in a nonempty open neighborhood of the boundary of $r_{N}\left(\underline{W}_{N} \cup W_{N}\right)$ and which contains only a finite number of fixed points all of which are in $r_{N}\left(\underline{W}_{N-1} \cup W_{N}\right)$. Pulling this flow back to $M$ we obtain a piecewise smooth flow $F_{N}: M \times I_{N} \rightarrow M$ which is ordinary except for a finite number of fixed points which are in the interior of the $m$-cells $B_{0}^{m}, \ldots, B_{N-1}^{m}$.

Proceeding inductively we obtain a piecewise smooth flow $F_{1}: M \times I_{1} \rightarrow M$ which is ordinary except for a finite number of fixed points which are in the interior of $B_{0}^{m}$.

For a proof of the following lemma see [10].

4.9. LEMMA. Let $D^{m}$ be the closed unit disc in $R^{m}$ with boundary $S^{m-1}$ and let s: $D^{m} \rightarrow T D^{m}$ be a smooth vector field on $D^{m}$ whose only zeroes occur at finitely many points $x_{k} \in D^{m}-S^{m-1}$. If $\sum i\left(s, x_{k}\right)=0$ then there is a nowhere vanishing vector field $s^{\prime}: D^{m} \rightarrow T D^{m}$ which agrees with $s$ in a neighborhood of $S^{m-1}$.

Let $D$ be a closed disc interior to $B_{0}^{m}$ which contains all the fixed points of $F_{1}$. Since $\chi(M)=0$ we may use Lemma 4.9 to alter the vector field on $D$ generated by $F_{1}: M \times I_{1} \rightarrow M$ to obtain a nowhere vanishing vector field on $D$ and then integrate to obtain a nonvanishing piecewise smooth flow $F_{0}: M \times I_{0} \rightarrow M$ on $M$. Q.E.D.

Lemma 4.9 is also the key lemma in proving the classical smooth version of Theorem 4.8 (see [10]); in fact the proof of Theorem 4.8 closely parallels the proof of this classical result.

5. Riemannian metrics. In this section Riemannian metrics are defined on simplicial spaces. We will show that the topology on a connected simplicial space induced by such a metric is the topology of the simplicial space.

5.1. Definition. The Whitney sum $\xi^{\prime} \oplus \xi^{\prime \prime}: E^{\prime} \oplus E^{\prime \prime} \rightarrow X$ of the simplicial bundles $\xi^{\prime}: E^{\prime} \rightarrow X$ and $\xi^{\prime \prime}: E^{\prime \prime} \rightarrow X$ is the simplicial bundle whose total space $E^{\prime} \oplus E^{\prime \prime}$ is the subspace of $E^{\prime} \times E^{\prime \prime}$ consisting of all those $\left(v^{\prime}, v^{\prime \prime}\right)$ for which $\xi^{\prime}\left(v^{\prime}\right)=\xi^{\prime \prime}\left(v^{\prime \prime}\right)$, whose projection $\xi^{\prime} \oplus \xi^{\prime \prime}$ is $\left(\xi^{\prime} \oplus \xi^{\prime \prime}\right)\left(v^{\prime}, v^{\prime \prime}\right)=\xi^{\prime}\left(v^{\prime}\right)$ $=\xi^{\prime \prime}\left(v^{\prime \prime}\right)$, whose fiber at $x \in X$ is $\left(\xi^{\prime} \oplus \xi^{\prime \prime}\right)^{-1} x=\left(\xi^{\prime}\right)^{-1} x \oplus\left(\xi^{\prime \prime}\right)^{-1} x$, and:

(1) for every simplicial chart $\left(U_{\alpha}, \psi_{\alpha}\right)$ on $X$ whose structure fiber $F_{\alpha}$ 
$=F_{\alpha}^{\prime} \oplus F_{\alpha}^{\prime \prime}$ and corresponding structure map $\phi_{\alpha}: U_{\alpha} \times F_{\alpha} \rightarrow\left(\xi^{\prime} \oplus \xi^{\prime \prime}\right)^{-1} U$ is $\phi_{\alpha}=\phi_{\alpha}^{\prime} \oplus \phi_{\alpha}^{\prime \prime}$, and

(2) for simplicial charts $\left(U_{\alpha}, \psi_{\alpha}\right)$ and $\left(U_{\beta}, \psi_{\beta}\right)$ on $X$ for which $U_{\alpha} \subseteq U_{\beta}$, whose structure map $l_{\beta \alpha}=l_{\beta \alpha}^{\prime} \oplus l_{\beta \alpha}^{\prime \prime}$.

A Riemannian metric on the $n$-corner $s^{n}$ is a smooth, symmetric, bilinear, positive definite map $\langle-,-\rangle: T s^{n} \oplus T s^{n} \rightarrow R$.

5.2. Definition. A Riemannian metric on the simplicial space $X$ is a symmetric, bilinear, positive definite map $\langle-,-\rangle: T X \oplus T X \rightarrow R$.

If $\langle-,-\rangle$ is a Riemannian metric on the simplicial space $X$, then for every simplicial chart $\psi_{\alpha}: U_{\alpha} \rightarrow V_{\alpha}$ on $X$,

$$
\left\langle\left(\psi_{\alpha}\right)_{*}-,\left(\psi_{\alpha}\right)_{*}-\right\rangle: T V_{\alpha} \oplus T V_{\alpha} \rightarrow R
$$

is a Riemannian metric on $V_{\alpha},\left(\psi_{\alpha}\right)_{*}$ the Jacobian of $\psi_{\alpha}$.

The existence of Riemannian metrics on simplicial spaces follows from the local construction of the tangent objects of simplicial spaces and the fact that piecewise smooth partitions of unity (subordinate to covers by small open subsets) can be defined on simplicial spaces.

A smooth curve $\gamma=\left(\gamma_{k}\right):[a, b] \rightarrow s^{n}$ in $s^{n}$ is a map for which each component function $\gamma_{k}$ of $\gamma$ is smooth on $(a, b)$, and for which the one-sided limits at $a$ and $b$ of all the derivatives of each $\gamma_{k}$ are finite; the tangent vector $T_{\gamma}\left(t_{0}\right)$ of $\gamma$ at $t_{0} \in[a, b]$ is

$$
T_{\gamma}\left(t_{0}\right)=\gamma_{*}\left((d / d t)_{t_{0}}\right)=\Sigma\left(d \gamma_{k} / d t\right)_{\mid t_{0}}\left(\partial / \partial x_{k}\right)_{\gamma\left(t_{0}\right)} .
$$

5.3. Defintion. A piecewise smooth curve $\gamma:[a, b] \rightarrow X$ in the simplicial space $X$ is a map for which there is a finite partition $a=c_{0} \leqslant c_{1} \leqslant \cdots \leqslant c_{n}=b$ of $[a, b]$ and, for each $k=0, \ldots, n-1$, a simplicial chart $\psi_{\alpha}: U_{\alpha} \rightarrow V_{\alpha}$ on $X$ with $\gamma\left(\left[c_{k}, c_{k+1}\right]\right) \subseteq U_{\alpha}$ and

$$
\psi_{\alpha} \circ \gamma:\left[c_{k}, c_{k+1}\right] \rightarrow V_{\alpha}
$$

a smooth curve. If $X$ is equipped with a Riemannian metric $\langle-,-\rangle$ and $|-|$ is the associated norm, then the length $L(\gamma)$ of $\gamma$ is

$$
L(\gamma)=\sum_{k=0}^{n-1} L\left(\gamma_{k}\right)=\sum_{k=0}^{n-1} \int_{c_{k}}^{c_{k+1}} T_{\gamma_{k}}(t) d t
$$

where $\gamma_{k}=\gamma_{\left[\left[c_{k}, c_{k+1}\right]\right.}$, and $T_{\gamma_{k}}(t)=\left(\gamma_{k}\right)_{*}\left((d / d t)_{t}\right)$.

The length $L(\gamma)$ of the piecewise smooth curve $\gamma:[a, b] \rightarrow X$ is clearly independent of the partition of $[a, b]$. Furthermore it follows from the chain rule that $L(\gamma)$ is independent of the parametrization of $\gamma$.

5.4. THEOREM. If $X$ is a connected simplicial space with Riemannian metric 
$\langle-,-\rangle$ then the map $d: X \times X \rightarrow R$ given by

$$
d(p, q)=\inf \{L(\gamma): \gamma \text { is a piecewise smooth curve from } p \text { to } q\}
$$

is a metric on $X$, and the metric topology on $X$ is the topology of the simplicial space.

Proof. The first assertion is immediate. The second assertion follows from the following result (see [13]).

5.5. LEMMA. Let $U$ and $W$ be small open neighborhoods of $x_{0} \in X$ which have compact closure and for which $W \subseteq U$. and let $u$ be a coordinate system on $U$ with origin $x_{0}$. If $|-|$ is the norm associated to the inner product $\langle-,-\rangle$ on $U$, and if $|-|_{0}$ is the norm associated to the inner product $\langle-,-\rangle_{0}$ on $U$ given by

$$
\left\langle\left(\partial / \partial u_{j}\right)_{x},\left(\partial / \partial u_{k}\right)_{x}\right\rangle_{0}=\delta_{j k}
$$

$\left(\delta_{j k}\right.$ the Kronecker delta) then there are positive real constants $m$ and $M$ for which

$$
m \cdot|-|_{0} \leqslant 1-1 \leqslant M \cdot|-|_{0}
$$

on $W$; consequently $m L_{0}(\gamma) \leqslant L(\gamma) \leqslant M L_{0}(\gamma)$ for any piecewise smooth curve $\gamma:[a, b] \rightarrow W$.

Proof (of Lemma 5.5). Let $V$ be the real vector space on generators $\partial / \partial u_{k}, u_{k} \in u$, and let

$$
S U=\left\{(x, v) \in U \times V:|(x, v)|_{0}=1 \text { and }(x, v) \in T U\right\} .
$$

Define $f: S U \rightarrow R$ by $f(x, v)=|(x, v)| ; f$ is positive and continuous. Since $W$ is compact there are positive constants $m$ and $M$ for which $m \leqslant f \leqslant M$ on $S W=\{(x, v) \in S U: x \in W\}$.

Now if $(x, v) \in T W$ and $v \neq 0$, let $\underline{v}=v /\left|\left(x_{0}, v\right)\right|_{0}=v /|(x, v)|_{0}$. Then

$$
m|(x, v)|_{0} \leqslant|(x, v)|_{0} f(x, \underline{v}) \leqslant M|(x, v)|_{0} .
$$

The conclusion follows since

$$
\begin{aligned}
|(x, v)|_{0} f(x, \underline{v}) & =|(x, v)|_{0}|(x, \underline{v})| \\
& =|(x, v)|_{0}\left(|(x, v)| /|(x, v)|_{0}\right) \\
& =|(x, v)| .
\end{aligned}
$$

(If $v=0$, then $m|(x, v)|_{0}=|(x, v)|=M|(x, v)|_{0}$.) Q.E.D.

6. A Gauss-Bonnet formula for polyhedral manifolds. In this section we give an integral formula for the Euler characteristic of a closed, oriented, evendimensional polyhedral manifold. As a consequence of this formula we obtain 
a representation for the Euler class of such a polyhedral manifold in terms of piecewise smooth forms. This integral formula brings together everything which has been done in this paper.

Let us first recall the following construction of smooth Euler classes (see [2], [9], and [12]): Let $\underline{M}$ be a smooth manifold (with or without boundary), let $A(M)$ be the ring of smooth functions on $\underline{M}$, and let $\left(\Lambda^{*} E(M), d(\underline{M})\right)$ be the smooth de Rham complex of $M$. If $\xi: E \rightarrow M$ is a smooth, oriented, real $2 n$ plane vector bundle over $\underline{M}$, let $\Gamma(\underline{M}, \xi)$ denote the $A(\underline{M})$-module of global smooth sections of $\xi$. A connexion on $\xi$ is a real linear map

$$
D: \Lambda^{*} E(M) \underset{A(M)}{\otimes} \Gamma(\underline{M}, \xi) \rightarrow \Lambda^{*} E(\underline{M}) \underset{A(M)}{\otimes} \Gamma(\underline{M}, \xi)
$$

of degree +1 for which $D(\theta \otimes s)=d \theta \otimes s+(-1)^{p} \theta \otimes D s$ for $\theta \in \Lambda^{p} E(\underline{M})$; the curvature $K$ of $D$ is the $\Lambda^{*} E(M)$-linear map $K=D \circ D$. A Riemannian metric on $\xi$, which may be considered as an inner product

$$
\langle-,-\rangle: \Gamma(\underline{M}, \xi) \otimes_{A(\underline{M})} \Gamma(\underline{M}, \xi) \rightarrow A(\underline{M})
$$

induces an inner product

$$
\langle-,-\rangle:\left(\Lambda^{*} E(M) \otimes \Gamma(\underline{M}, \xi)\right) \underset{\Lambda^{*} E(M)}{\otimes}\left(\Lambda^{*} E(\underline{M}) \otimes \Gamma(\underline{M}, \xi)\right) \rightarrow \Lambda^{*} E(\underline{M}),
$$

and the connexion $D$ on $\xi$ is metric with respect to the inner product $\langle-,-\rangle$ on $\xi$ iff

$$
\left\langle D s^{\prime}, s^{\prime \prime}\right\rangle+\left\langle s^{\prime}, D s^{\prime \prime}\right\rangle=d\left\langle s^{\prime}, s^{\prime \prime}\right\rangle
$$

for $s^{\prime}, s^{\prime \prime} \in \Gamma(M, \xi)$. If $\langle-,-\rangle$ is any metric on $\xi$ then the Euler class $e(\xi)$ of $\xi$ is

$$
e(\xi)=(-1)^{n(n-1) / 2} 4 \pi^{-n}\left[\operatorname{Tr}\left(* \circ \Lambda^{n} K\right)\right] \in H_{\mathrm{de} \operatorname{Rham}}^{2 n}(\underline{M}),
$$

*: $\Lambda^{*} E(M) \rightarrow \Lambda^{*} E(M)$ denoting the Hodge star operator, and $K$ the curvature of any metric connexion $D$ on $\xi$. (It is not difficult to show that this definition of $e(\xi)$ is independent of the particular metric $\langle-,-\rangle$ and metric connexion $D$ chosen on $\xi$.) If $M$ is even-dimensional, the Euler class $e(\underline{M})=e(\tau(\underline{M})), \tau(\underline{M}): T \underline{M} \rightarrow \underline{M}$ the tangent bundle of $\underline{M}$.

In the following result we let $e(\xi)$ denote the $2 n$-form

$$
e(\xi)=(-1)^{n(n-1) / 2} 4 \pi^{-n} \operatorname{Tr}\left(* \circ \Lambda^{n} K\right) \in \Lambda^{2 n} E(\underline{M}),
$$

$K$ the curvature of any metric connexion $D$ on $\xi$.

6.1. THEOREM. Let $M$ be a smooth $2 n$-dimensional manifold with boundary $\partial M$. Let $\xi: E \rightarrow M$ be a smooth, oriented, real 2 -plane vector bundle over $\underline{M}$ with metric $\langle-,-\rangle$ and corresponding norm $|-|$. If $\underline{E}_{0}=\{v \in E:|v|>0\}$ and 
$\xi_{0}: E_{0} \rightarrow M$ is the projection induced by $\xi$, then the inclusion $E_{0} \rightarrow E$ induces a smooth nonzero section $s_{0}$ of $\xi_{0}^{\prime} \xi: E\left(\xi_{0}^{\prime} \xi\right) \rightarrow E_{0}$. Furthermore there is a smooth $(2 n-1)$-form $\eta(\xi)$ defined on $\underline{E}_{0}$ for which:

(1) $\xi_{0}^{*} e(\xi)=d \eta(\xi)$,

(2) if $j: S^{2 n}\left(\xi^{-1}\left(x_{0}\right)\right) \rightarrow E_{0}$ is the inclusion of the unit sphere of $\xi^{-1}\left(x_{0}\right)$ into $E_{0}$, then $-j^{*} \eta(\xi)$ is the orientation class of $S^{2 n}\left(\xi^{-1}\left(x_{0}\right)\right)$, and

(3) a smooth nonzero section s: $\partial \underline{M} \rightarrow E_{0} \subseteq \underline{E}$ of $\xi$ defined on $\partial M$ extends to a smooth nonzero section of $\xi$ defined on $M$ iff

$$
\int_{M} e(\xi)-\int_{\partial M} s^{*} \eta(\xi)=0 .
$$

Proof. The form $\eta(\xi)$ is defined by

$$
\eta(\xi)=\pi^{-n} \sum_{p=0}^{n-1}(-1)^{p}\left(1 \cdot 3 \cdots(2 n-2 p-1) p ! 2^{n+p}\right)^{-1} \operatorname{Tr} \Phi_{p}
$$

where, for $p=0,1, \ldots, n-1$,

$$
\Phi_{p}: \Lambda^{*} E(\underline{M}) \underset{A(M)}{\otimes} \Lambda^{p} \Gamma(\underline{M}, \xi) \rightarrow \Lambda^{*} E(\underline{M}) \underset{A(M)}{\otimes} \Lambda^{p} \Gamma(\underline{M}, \xi)
$$

is given on generators by

$$
\Phi_{p}\left(e_{1} \wedge \cdots \wedge e_{p}\right)=*\left(s_{0} \wedge\left(D s_{0}\right)^{2 n-2 p-1} \wedge \Lambda^{p} K\left(e_{1} \wedge \cdots \wedge e_{p}\right)\right) .
$$

The third part of the conclusion follows from the fact that if $s$ is a smooth section of $\xi$ which is nonzero on $\partial M$ and which has only a finite number of isolated zeroes then

$$
\int_{M} e(\xi)-\int_{\partial M} s^{*} \eta(\xi)=\Sigma i\left(s, x_{k}\right)
$$

where the summation is taken over the set $\left\{x_{k}\right\}$ of zeroes of $s$ and $i\left(s, x_{k}\right)$ is defined as follows: Let $D^{2 n}(\varepsilon)$ be a closed ball of radius $\varepsilon$ about $x_{k}$ relative to a local coordinate system in a neighborhood of $x_{k}$ with origin $x_{k}$, and let $\phi: \xi^{-1}\left(D^{2 n}(\xi)\right) \rightarrow \xi^{-1}\left(x_{k}\right)$ be a retraction which is an isomorphism on each fiber. Then for small enough positive $\delta<\varepsilon$,

$$
\phi \circ s: \partial D^{2 n}\left(\delta_{0}\right) \rightarrow \xi^{-1}\left(x_{k}\right)-\{0\}
$$

for every positive $\delta_{0}<\delta$; the index $i\left(s, x_{k}\right)$ is defined as the degree of $\phi \circ s$. (If $F: M \times R \rightarrow M$ is a smooth flow on $\underline{M}$ with an isolated fixed point at $x_{0}$ and $s$ is the smooth vector field on $M$ generated by $F$ then $i\left(s, x_{0}\right)$ agrees with the index $i\left(s, x_{0}\right)$ as defined immediately before Lemma 4.2.) Q.E.D.

In particular Theorem 6.1 is valid for $M$ the closed unit $2 n$-ball $D^{2 n} \subseteq R^{2 n}$ 
and $\xi$ the tangent bundle $\tau(\underline{M}): T \underline{M} \rightarrow M$ of $M=D^{2 n}$. But moreover the analogous result is valid for the $2 n$-cell $B^{2 n}=[-1,1]^{2 n} \subseteq R^{2 n}$ and $\xi$ the tangent object (= tangent vector bundle) $\tau\left(B^{2 n}\right): T B^{2 n} \rightarrow B^{2 n}$ of $B^{2 n}$ : The Euler class $e\left(B^{2 n}\right)=e\left(\tau\left(B^{2 n}\right)\right)$ of $B^{2 n}$ may be defined exactly as in the smooth case, and furthermore we have the following result.

6.2. LemMa. Let $\tau\left(B^{2 n}\right): T B^{2 n} \rightarrow B^{2 n}$ be the tangent object of the oriented $2 n$ cell $B^{2 n}$ with metric $\langle-,-\rangle$ and corresponding norm $|-|$. If $T B_{0}^{2 n}=\left\{v \in T B^{2 n}\right.$ : $|v|>0\}$ and $\tau\left(B^{2 n}\right)_{0}: T B_{0}^{2 n} \rightarrow B^{2 n}$ is the projection induced by $\tau\left(B^{2 n}\right)$, then the inclusion $T B_{0}^{2 n} \rightarrow T B^{2 n}$ induces a smooth nonzero section $s_{0}$ of

$$
\tau\left(B^{2 n}\right)_{0}^{!} \tau\left(B^{2 n}\right): E\left(\tau\left(B^{2 n}\right)_{0}^{!} \tau\left(B^{2 n}\right)\right) \rightarrow T B_{0}^{2 n} .
$$

Furthermore there is a smooth $(2 n-1)$-form $\eta\left(B^{2 n}\right)$ defined on $T B_{0}^{2 n}$ for which:

(1) $\tau\left(B^{2 n}\right)_{0}^{*} e\left(B^{2 n}\right)=d \eta\left(B^{2 n}\right)$,

(2) if $j: S^{2 n}\left(\tau\left(B^{2 n}\right)^{-1} x_{0}\right) \rightarrow T B_{0}^{2 n}$ is the inclusion of the unit sphere of $\tau\left(B^{2 n}\right)^{-1} x_{0}$ into $T B_{0}^{2 n}$ then $-j^{*} \eta\left(B^{2 n}\right)$ is the orientation class of $S^{2 n}\left(\tau\left(B^{2 n}\right)^{-1} x_{0}\right)$, and

(3) a piecewise smooth nonzero section $s: \partial B^{2 n} \rightarrow T B_{0}^{2 n} \subseteq T B^{2 n}$ of $\tau\left(B^{2 n}\right)$ defined on $\partial B^{2 n}$ extends to a smooth nonzero section of $\tau\left(B^{2 n}\right)$ defined on $B^{2 n}$ iff

$$
\int_{B^{2 n}} e\left(B^{2 n}\right)-\int_{\partial B^{2 n}} s^{*} \eta\left(B^{2 n}\right)=0 .
$$

Proof. The proof of this result is identical to the proof of Theorem 6.1. Q.E.D.

Now let $M$ be a closed, oriented, $2 n$-dimensional polyhedral manifold. We obtain the integral formula for the Euler characteristic of $M$ as follows: Let $F: M \times I \rightarrow M$ be any piecewise smooth flow on $M$ which has only a finite number of fixed points which are all interior to $2 n$-cells of $M$; the existence of such flows is insured by Corollary 4.6.

6.3. LEMMA. Let $B_{\alpha}^{2 n}$ be a $2 n$-cell of $M$ and let $s_{\alpha}: B_{\alpha}^{2 n} \rightarrow T B_{\alpha}^{2 n}$ be the section of the tangent object (= tangent vector bundle) $\tau\left(B^{2 n}\right)$ of $B_{\alpha}^{2 n}$ induced by the

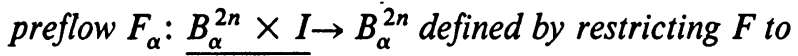

$$
B_{\alpha}^{2 n} \times I=\left\{(x, t) \in B_{\alpha}^{2 n} \times I: F(x, t) \in B_{\alpha}^{2 n}\right\} .
$$

Then $s_{\alpha}$ is nonzero on the boundary $\partial B_{\alpha}^{2 n}$ of $B_{\alpha}^{2 n}$.

Proof. Let us first observe that if $U$ and $W$ are small open subsets of the $n$-corner $s^{n}$ which have compact closure and for which the closure of $W$ is contained in $U$, and $F: \underline{W \times R} \rightarrow U$ is a preflow on $W$ for which

$$
\left(\Sigma\left(\partial F_{k}(x, t) / \partial t\right)_{\mid t=0}\left(\partial / \partial x_{k}\right)\right) x_{0}=0
$$


then $\left\{x_{0}\right\} \times R \subseteq \underline{W \times R}$ and $F\left(x_{0}, t\right)=x_{0}$ for all $t \in R$ : According to Lemma 3.2 the preflow $F$ can be reconstructed from the piecewise smooth functions $f_{k}=\left(\partial F_{k}(x, t) / \partial t\right)_{\mid t=0}$ by first extending the functions $f_{k}$ to functions $\underline{f}_{k}$ defined on an open subset of $R^{n}$ which has compact closure, applying Picard's method, and then restricting the resulting flow $F$ to $W \times R$. However if $f_{k}\left(x_{0}\right)=0$ for each $k$ then every flow $F$ on $R^{n}$ constructed in this manner will have a fixed point at $x_{0}$. The desired conclusions now follow from the construction of the preflow $F$ from the flow $F$.

Now let $x_{0}$ be a point on the $(2 n-1)$-skeleton of $M$ (hence an ordinary point of the piecewise smooth flow $F: M \times I \rightarrow M),(U, \Psi)$ a packet on $M$ for which $U$ is a small open neighborhood of $x_{0}$ (with compact closure) and $u$ a coordinate system on $(U, \Psi)$. Let $W$ be a small open neighborhood of $x_{0}$ for which the closure of $W$ is contained in $U$ and $J \subseteq I$ an open interval containing the origin for which $F: W \times J \rightarrow U$. If $\psi_{\alpha}: U_{\alpha} \rightarrow V_{\alpha} \subseteq s_{\alpha}^{2 n}$ is any simplicial chart of $\Psi$ then the restriction $F_{\alpha}$ of $F$ to

$$
\underline{\left(W \cap U_{\alpha}\right) \times J}=\left\{(x, t) \in\left(W \cap U_{\alpha}\right) \times J: F(x, t) \in U_{\alpha}\right\}
$$

is a preflow on $W \cap U_{\alpha}$. If $\left(\left(\partial\left(u_{\alpha}^{k} \circ F_{\alpha}\right) / \partial t\right)_{\mid t=0}\right) x_{0}=0$ for each $k$ then $\left\{x_{0}\right\} \times J \subseteq\left(W \cap U_{\alpha}\right) \times J$ and $F\left(x_{0}, t\right)=x_{0}$ for each $t \in J$, contradicting the fact that $x_{0}$ is an ordinary point of $F$. Q.E.D.

Now a brief digression.

Recall (see [11]) that there is a natural morphism $\rho$ called restriction from the de Rham complex $\left(\Lambda^{*} E\left(s^{n}\right), d\left(s^{n}\right)\right)$ of the $n$-corner $s^{n}$ to the de Rham complex $\left(\Lambda^{*} E\left(s^{m}\right), d\left(s^{m}\right)\right)$ of the face $s^{m}$ of $s^{n}$ defined by

$$
\rho(\theta)=\rho\left(\sum f_{k} d x_{k_{1}} \cdots d x_{k_{p}}\right)=\sum\left(f_{k \mid s} m\right) d\left(x_{k_{1} \mid s} m\right) \cdots d\left(x_{k_{p} \mid s} m\right) .
$$

This morphism induces a morphism, again called restriction, from the de Rham complex $\left(\Lambda^{*} E(X), d(X)\right)$ of any simplicial space $X$ to the de Rham complex $\left(\Lambda^{*} E\left(X^{m}\right), d\left(X^{m}\right)\right)$ of the $m$-skeleton $X^{m}$ of $X$.

6.4. LEMMA. Any piecewise smooth p-form $\theta$ defined on the $(n-1)$-skeleton of an arbitrary simplicial space $X$ extends to a piecewise smooth $p$-form $\underline{\theta}$ defined on the $n$-skeleton of $X$ (i.e., $\underline{\theta}$ restricts to $\theta)$.

Proof. Since piecewise smooth partitions of unity subordinate to covers of $X$ by small open subsets exist, it suffices to prove this lemma locally. Proving the local version of this lemma reduces to proving this lemma in the special case $X=s^{n}$.

If $f$ is a piecewise smooth function defined on the boundary of $s^{n}$ then an extension $f \in A\left(s^{n}\right)$ of $f$ may be defined as follows: For each $j$-tuple $k=\left(k_{1}, \ldots, k_{j}\right), j=1, \ldots, n$, define the projection $\pi_{k}$ from $s^{n}$ to the boundary of $s^{n}$ by taking each $x \in s^{n}$ to the point whose coordinates in the $k$-slots 
are zero, and whose coordinates are otherwise unchanged; then $\underline{f}$ $=\Sigma_{k}(-1)^{k+1}\left(f \circ \pi_{k}\right)$ where the summation is taken over all $j$-tuples $\underline{k}$, $j=1, \ldots, n$, and where for each $j$-tuple $k,(-1)^{k+1}=(-1)^{j+1}$.

Given $\theta=\sum f_{k} d x_{k_{1}} \cdots d x_{k_{p}}$ defined on the boundary of $s^{n}, \underline{\theta}$ may be defined by $\Sigma f_{k} d x_{k_{1}} \cdots d x_{k_{p}}$. Q.E.D.

Now again let $M$ be a closed, oriented, $2 n$-dimensional polyhedral manifold. Let $\langle-,-\rangle$ be any Riemannian metric on $M$. Each cell $B_{\alpha}^{2 n}$ of $M$ inherits an orientation and a Riemannian metric naturally from $M$, and hence we may construct the smooth $2 n$-form

$$
O\left(B_{\alpha}^{2 n}\right)=e\left(B_{\alpha}^{2 n}\right)-d\left(B_{\alpha}^{2 n}\right) \underline{s_{\alpha}^{*} \eta\left(B_{\alpha}^{2 n}\right)} \in \Lambda^{2 n} E\left(B_{\alpha}^{2 n}\right)
$$

on $B_{\alpha}^{2 n}$ where $s_{\alpha}$ is as in Lemma 6.3.

6.5. LEMMA. The forms $O\left(B_{\alpha}^{2 n}\right)$, for $B_{\alpha}^{2 n}$ a $2 n$-cell of $M$, define a global closed piecewise smooth 2 -form $e(M)$ on $M$.

Proof. The fact that the forms $O\left(B_{\alpha}^{2 n}\right)$ define a global piecewise smooth $2 n$ form $e(M)$ on $M$ follows immediately from the definition of a piecewise smooth form on $M$. The fact that $e(M)$ is closed follows from the fact that there are no nontrivial piecewise smooth $(2 n+1)$-forms on $M$. Q.E.D.

The definition of $e(M)$ depends a priori on many choices (such as the piecewise smooth flow $F: M \times I \rightarrow M$ on $M$, Riemannian metric, metric connexions, and extensions). It is not difficult to verify directly that the definition of $e(M)$ is independent of all such choices. However the same result follows immediately from the following result which is the main result of this paper. The proof of this theorem naturally parallels Chern's intrinsic proof of the Gauss-Bonnet formula for closed Riemannian manifolds (see [5]).

6.6. Theorem (Polyhedral Gauss-BonNET). If $M$ is a closed, oriented, $2 n$ dimensional polyhedral manifold and $e(M) \in \Lambda^{2 n} E(M)$ is the piecewise smooth $2 n$-form on $M$ constructed above then

where $\chi(M)$ is the Euler characteristic of $M$.

$$
\int_{M} e(M)=\chi(M)
$$

Proof. Using Stokes's theorem,

$$
\begin{aligned}
\int_{M} e(M) & =\Sigma \int_{B_{\alpha}^{2 n}} O\left(B_{\alpha}^{2 n}\right) \\
& =\Sigma\left(\int_{B_{\alpha}^{2 n}}\left(e\left(B_{\alpha}^{2 n}\right)-d\left(B_{\alpha}^{2 n}\right) \underline{\left.s_{\alpha}^{*} \eta\left(B_{\alpha}^{2 n}\right)\right)}\right)\right. \\
& =\Sigma\left(\int_{B_{\alpha}^{2 n}} e\left(B_{\alpha}^{2 n}\right)-\int_{\partial B_{\alpha}^{2 n}} s^{*} \eta\left(B_{\alpha}^{2 n}\right)\right)
\end{aligned}
$$


Using Lemma 6.2 and Lemma 6.3,

$$
\int_{M} e(M)=\sum i\left(s_{\alpha}, x_{k}\right)
$$

Using Lemma $4.2, \int_{M} e(M)=\sum i\left(F, x_{k}\right)$, so that, by Theorem 4.3, $\int_{M} e(M)$ $=\chi(M)$. Q.E.D.

Theorem 6.6 is closely related to a similar integral formula given by Allendoerfer and Weil (see [1]) for the Euler characteristic of a smooth manifold with piecewise smooth boundary. This latter result is obtained as follows: one first proves a generalized Gauss-Bonnet formula for a piece of a Riemannian manifold isometrically embedded in Euclidean space, and then pieces manifolds and forms together; the generalized formula is proved by first verifying the formula in a special case (namely the case of an embedded curved simplex), and then proceeding to the general case by using the fact that the additivity of angles implies an additivity property for integrals.

6.7. REMARK. As a result of Theorem 6.6, the Euler class $e(M)$ $\in H_{\mathrm{de} \mathrm{Rham}}^{2 n}(M)$ of the closed, oriented, $2 n$-dimensional polyhedral manifold $M$ is the class described by the piecewise smooth $2 n$-form $e(M) \in \Lambda^{2 n} E(M)$ constructed above.

6.8. REMARK. If $\underline{M}$ is a closed, oriented, $2 n$-dimensional smooth manifold, $e(\underline{M})$ is the Euler class of $\underline{M}$, and $T: M \rightarrow \underline{M}$ is a triangulation of $\underline{M}$ by a polyhedral manifold $M$, then $T^{*} e(\underline{M})=e(M)$.

\section{REFERENCES}

1. C. B. Allendoerfer and André Weil, The Gauss-Bonnet theorem for Riemannian polyhedra, Trans. Amer. Math. Soc. 53 (1943), 101-129. MR 4, 169.

2. Raoul Bott and S. S. Chern, Hermitian vector bundles and the equidistribution of the zeroes of their holomorphic sections, Acta Math. 114 (1965), 71-112. MR 32 \#3070.

3. R. F. Brown, The Lefshetz fixed point theorem, Scott, Foresman, Glenview, Ill., 1971. MR 44 \# 1023.

4. S. S. Cairns, Triangulated manifolds which are not Brouwer manifolds, Ann. of Math. (2) 41 (1940), 792-795. MR 2, 74.

5. S. S. Chern, A simple intrinsic proof of the Gauss-Bonnet formula for closed Riemannian manifolds, Ann. of Math. (2) 45 (1944), 747-752. MR 6, 106.

6. E. A. Coddington and Norman Levinson, Theory of ordinary differential equations, McGrawHill, New York, 1955. MR 16, 1022.

7. H. Hopf, Vektorfelder in n-dimensionalen Mannigfaltigkeiten, Math. Ann. 96 (1927), 225-250.

8. J. Milnor, Lectures on characteristic classes, Princeton University, 1958 (mimeographed notes).

9. Howard Osborn, Representations of Euler classes, Proc. Amer. Math. Soc. 31 (1972), 340-346. MR 45 \#9349.

10. _ Gauss-Bonnet in DIFF and in PL, Univ. of Illinois, 1972 (mimeographed notes).

11. M. Penna, Differential geometry on simplicial spaces, Trans. Amer. Math. Soc. 214 (1975), 303-323. 
12. Differential geometry on simplicial manifolds, Dissertation, Univ. of Illinois, Urbana-Champaign, III., 1974.

13. Michael Spivak, $A$ comprehensive introduction to differential geometry. Vols. I, II, Publish or Perish, Waltham, Mass., 1970. MR 42 \#2369; 6726.

14. Hassler Whitney, On the topology of differentiable manifolds, Lectures in Topology, Univ. of Michigan Press, Ann Arbor, Mich., 1941. MR 3, 133.

Departmant of Mathematics, Indiana-Purdue University, Indianapolis, Indiana 46205 